\title{
Many new variable stars discovered in the core of the globular cluster NGC 6715 (M 54) with EMCCD observations ${ }^{\star}, \star \star$
}

\author{
R. Figuera Jaimes ${ }^{1,2}$, D. M. Bramich ${ }^{3}$, N. Kains ${ }^{6}$, J. Skottfelt ${ }^{4,5}$, U. G. Jørgensen ${ }^{5}$, K. Horne ${ }^{1}$, M. Dominik ${ }^{1, \star \star \star}$,

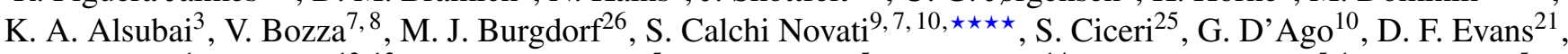 \\ P. Galianni ${ }^{1}$, S.-H. Gu ${ }^{12,13}$, K. B. W Harpsøe ${ }^{5}$, T. Haugbølle ${ }^{5}$, T. C. Hinse ${ }^{14}$, M. Hundertmark ${ }^{5,1}$, D. Juncher ${ }^{5}$, \\ E. Kerins ${ }^{29}$, H. Korhonenn $24,15,5$, M. Kuffmeier ${ }^{5}$, L. Mancini ${ }^{11}$, N. Peixinho ${ }^{27,28}$, A. Popovas ${ }^{5}$, M. Rabus ${ }^{16,11}$, \\ S. Rahvar ${ }^{17}$, G. Scarpetta ${ }^{10,7,8}$, R. W. Schmidt ${ }^{18}$, C. Snodgrass ${ }^{19,20}$, J. Southworth ${ }^{21}$, D. Starkey ${ }^{1}$, R. A. Street ${ }^{22}$, \\ J. Surdej ${ }^{23}$, R. Tronsgaard ${ }^{30}$, E. Unda-Sanzana ${ }^{27}$, C. von Essen ${ }^{30}$, X.-B. Wang ${ }^{12,13}$, and O. Wertz ${ }^{23}$ \\ (The MiNDSTEp Consortium) \\ (Affiliations can be found after the references)
}

Received 6 May 2016 / Accepted 27 May 2016

\begin{abstract}
Context. We show the benefits of using electron-multiplying CCDs and the shift-and-add technique as a tool to minimise the effects of atmospheric turbulence, such as blending between stars in crowded fields, and to avoid saturated stars in the fields observed. We intend to complete, or improve on, the census of the variable star population in globular cluster NGC 6715.

Aims. Our aim is to obtain high-precision time-series photometry of the very crowded central region of this stellar system via the collection of better angular resolution images than has been previously achieved with conventional CCDs on ground-based telescopes.

Methods. Observations were carried out using the Danish 1.54-m telescope at the ESO La Silla observatory in Chile. The telescope is equipped with an electron-multiplying CCD that enables short-exposure-time images to be obtained (ten images per second) that were stacked using the shift-and-add technique to produce the normal-exposure-time images (minutes). The high precision photometry was performed via difference image analysis employing the DanDIA pipeline. We attempted automatic detection of variable stars in the field.

Results. We statistically analysed the light curves of 1405 stars in the crowded central region of NGC 6715 to automatically identify the variable stars present in this cluster. We found light curves for 17 previously known variable stars near the edges of our reference image (16 RR Lyrae and 1 semi-regular) and we discovered 67 new variables (30 RR Lyrae, 21 irregular (long-period type), 3 semi-regular, 1 W Virginis, 1 eclipsing binary, and 11 unclassified). Photometric measurements for these stars are available in electronic form through the Strasbourg Astronomical Data Centre.
\end{abstract}

Key words. atmospheric effects - instrumentation: high angular resolution - globular clusters: individual: NGC 6715 (M 54) methods: observational - stars: variables: general - stars: variables: RR Lyrae

\section{Introduction}

Galactic globular clusters are interesting stellar systems in astronomy since they are fossils of early Galaxy formation and evolution. This makes them excellent laboratories in a wide range of topics from stellar evolution to cosmology, from observations to theory.

NGC 6715 (M 54) was discovered on July 24, 1778 by Charles Messier ${ }^{1}$. The cluster is in the Sagittarius dwarf spheroidal galaxy at a distance of $26.5 \mathrm{kpc}$ from our Sun and $18.9 \mathrm{kpc}$ from our Galactic centre. It has a metallicity $[\mathrm{Fe} / \mathrm{H}]=-1.49$ dex and a distance modulus $(m-M)_{V}=$ $17.58 \mathrm{mag}$. The magnitude of its horizontal branch is $V_{\mathrm{HB}}=$ 18.16 mag (2010 version Harris 1996). The stellar population and morphology of NGC 6715 have attracted the attention of several studies. Although NGC 6715's position is aligned with the central region of the Sagittarius dwarf spheroidal

\footnotetext{
* Based on data collected by MiNDSTEp with the Danish $1.54 \mathrm{~m}$ telescope at the ESO La Silla observatory.

$\star \star$ Full Table 1 is only available at the CDS via anonymous ftp to cdsarc.u-strasbg.fr (130.79.128.5) or via http://cdsarc.u-strasbg.fr/viz-bin/qcat?J/A+A/592/A120 $\star \star \star$ Royal Society University Research Fellow.

$\star \star \star \star$ Sagan visiting fellow.

1 http://messier. seds.org/m/m054.html
}

galaxy, recent studies have shown that this cluster is not the nucleus of the mentioned galaxy (Layden \& Sarajedini 2000; Majewski et al. 2003; Monaco et al. 2005; Bellazzini et al. 2008). Carretta et al. (2010) found that the metallicity spread in this cluster is intermediate between smaller-normal Galactic globular clusters and metallicity values associated with dwarf galaxies. It has a very peculiar colour-magnitude diagram with multiple main sequences, turnoff points, and an extended blue horizontal branch (HB) (Siegel et al. 2007; Milone 2015). Rosenberg et al. (2004) found that this cluster hosts a blue hook stellar population in its blue HB. It is thought that NGC 6715 might host an intermediate mass black hole (IMBH) in its centre (Ibata et al. 2009; Wrobel et al. 2011) as well.

Oosterhoff (1939) found that globular clusters can be classified into two groups based on the mean periods and the number ratios of their RR0 and RR1 stars. These are the Oosterhoff I (OoI) and Oosterhoff II (OoII) groups, and this is known as the Oosterhoff dichotomy. It was also found that the metallicity of the clusters plays an important role in this classification (Kinman 1959) and that it could be related with the formation history of the Galactic halo (see e.g. discussions in Lee \& Carney 1999; Catelan 2004, 2009; Smith et al. 2011; Sollima et al. 2014).

The first Oosterhoff classification for NGC 6715 was done by Layden \& Sarajedini (2000) by comparing the amplitudes and periods of 67 RR Lyrae in this cluster with the period-amplitude 
relation obtained for M3 (OoI) and M9 (OoII). NGC 6715 follows the relation defined by M3 and this implies that it is an OoI cluster. They also found that the mean periods of the RR Lyrae also agree with the OoI classification. Similarly, Sollima et al. (2010), in their study of variable stars in NGC 6715, discovered a new set of 80 RR Lyrae, and they used 95 RR0 and 33 RR1 (after excluding non-members or problematic stars) to find that this cluster shares some properties of both OoI and OoII clusters. For instance, the average period of its RR0 and RR 1 stars was found to be intermediate between the values for the OoI and OoII classifications. We note that Catelan (2009) also classified this cluster as an intermediate Oosterhoff type based on its position in the $\left\langle P_{\mathrm{RR} 0}\right\rangle$-metallicity diagram.

Several time-series photometric studies focused on the variable star population (Rosino 1952; Rosino \& Nobili 1958; Layden \& Sarajedini 2000; Sollima et al. 2010; Li \& Qian 2013), but owing to the high concentration of stars in its core, many variables have previously been missed because of blending. NGC 6715 is very massive and it is among the densest known globular clusters (Pryor \& Meylan 1993). As a result, NGC 6715 also caught our attention as an excellent candidate for our study of globular clusters using electronmultiplying CCDs (EMCCDs) and the shift-and-add technique (Skottfelt et al. 2013, 2015a; Figuera Jaimes et al. 2016).

Section 2 summarises the observations, the reduction, and photometric techniques employed. Section 3 explains the calibration applied to the instrumental magnitudes. Section 4 contains the technique used in the detection and extraction of variable stars. Sections 5 and 6 show the methodology used to classify variables and the colour-magnitude diagrams employed, respectively. Section 7 presents our results and Sect. 8 discusses the Oosterhoff classification of this cluster. Our conclusions are presented in Sect. 9.

\section{Data, reduction, and photometry}

EMCCDs, also known as low light level charge-coupled devices (L3CCD; see e.g. Smith et al. 2008; Jerram et al. 2001), are conventional CCDs that have an extended readout register where the signal is amplified by impact ionisation before they are read out. Hence the readout noise is negligible when compared to the signal and very high frame-rates become feasible (10-100 frames/s). This provides an opportunity to compensate for the blurring effect of the turbulence in the atmosphere. By shifting and adding the individual frames appropriately, it is possible to construct much higher resolution images than is possible using conventional CCD imaging from the ground. Furthermore, the dynamic range of the stacked images is greatly increased and the saturation of bright stars is therefore not an issue except for the very brightest stars in the sky. However, the main drawback with this technique is that the process, whereby the signal is amplified, also increases the photon noise component in the images by a factor of $\sqrt{2}$, when compared to conventinal CCD images. We also need to be aware that EMCCD imaging data need to be calibrated in a different way to conventional CCD data. Several studies where EMCCDs have been used can be found in the literature, e.g. high-resolution imaging of exoplanet host stars in the search for unseen companions (Southworth et al 2015; Ciceri et al. 2016; Bozza et al. 2016; Street et al. 2016; Evans et al. 2016) photometric and astrometric measurements of a pair of very close brown dwarfs (Mancini et al. 2015), and time-series photometry of crowded globular cluster cores aiming to complete the census of variable stars (Skottfelt et al. 2015a; Figuera Jaimes et al. 2016).

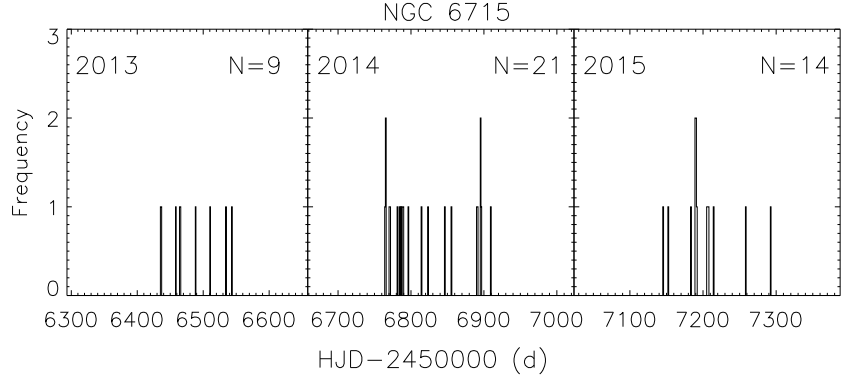

Fig. 1. Histograms with the number of observations per night for globular cluster NGC 6715. Left: 2013. Middle: 2014. Right: 2015.

The data presented in this paper are the result of EMCCD observations performed over three consecutive years: 2013, 2014, and 2015 in April to September each year. The $1.54 \mathrm{~m}$ Danish telescope at the ESO Observatory in La Silla, Chile was used with an Andor Technology iXon+897 EMCCD camera, which has a $512 \times 512$ array of $16 \mu \mathrm{m}$ pixels, giving a pixel scale of $\sim 0^{\prime \prime}$.09 per pixel and a total field of view of $\sim 45 \times 45 \mathrm{arcsec}^{2}$.

For this research, the EMCCD camera was set to work at a frame-rate of $10 \mathrm{~Hz}$ (ten frames per second) and an EM gain of $300 e^{-} /$photon. The camera was placed behind a dichroic mirror which works as a long-pass filter. Taking the mirror and the sensitivity of the camera into consideration, it is possible to cover the wavelength range from $650 \mathrm{~nm}$ to $1050 \mathrm{~nm}$. This is roughly a combination of SDSS $i^{\prime}+z^{\prime}$ filters (Bessell 2005). More details about the instrument can be found in Skottfelt et al. (2015b). The total exposure time employed for a single observation was ten minutes, which means that each observation is the result of shifting-and-adding 6000 exposures. The resulting point spread function (PSF) full width at half maximum (FWHM) in the reference image employed in the photometric reductions (see below) was $0.44^{\prime \prime}$, while the mean standard seeing calculated with the values reported at the observatory station during April to September 2013, 2014, and 2015 were $1.0^{\prime \prime}, 1.2^{\prime \prime}$, and $0.9^{\prime \prime}$, respectively.

In Fig. 1, histograms of the number of observations per night during each year are shown. Data in the left-hand panel correspond to 2013, data in the middle panel to 2014, and data in the right-hand panel to 2015 . We aimed to always take two observations per night, although it was not always possible because of weather conditions or time slots needed for other projects, as is the case of the monitoring of microlensing events carried out by the MiNDSTEp consortium.

Bias, flat-field, and tip-tilt corrections were performed using the procedures and algorithms described in Harpsøe et al. (2012). In particular, the tip-tilt correction allows high-resolution stacked images to be created, as described in detail in Skottfelt et al. (2015a), Figuera Jaimes et al. (2016). Briefly, the method uses the Fourier cross-correlation theorem where, for each bias- and flat-corrected exposure $I_{k}(i, j)$ within an observation, we calculate the cross-correlation image between $I_{k}(i, j)$ and an average of 100 randomly chosen exposures. The peak in the cross-correlation image gives the appropriate shift to correct the tip-tilt error. We then stack the shifted exposures according to batches grouped by image quality (a measure of image sharpness), to create ten-layer image cubes. The best quality layers from each cube (i.e. the sharpest layers) are extracted from all of the available observations to create a high-resolution reference image for subsequent difference image analysis (DIA). Finally, each ten-layer cube is stacked to create a single image that corresponds to each observation. 
Table 1. Time-series $I$-band photometry for all known and new variables in the field of view covered in globular cluster NGC 6715.

\begin{tabular}{|c|c|c|c|c|c|c|c|c|c|c|}
\hline $\begin{array}{l}\text { var } \\
\text { id } \\
(1)\end{array}$ & $\begin{array}{l}\text { Filter } \\
\text { (2) }\end{array}$ & $\begin{array}{c}\text { HJD } \\
\text { (d) } \\
(3) \\
\end{array}$ & $\begin{array}{c}M_{\text {std }} \\
(\mathrm{mag}) \\
(4)\end{array}$ & $\begin{array}{c}m_{\mathrm{ins}} \\
(\mathrm{mag}) \\
(5)\end{array}$ & $\begin{array}{c}\sigma_{m} \\
(\mathrm{mag}) \\
(6)\end{array}$ & $\begin{array}{c}f_{\mathrm{ref}} \\
\left(\mathrm{ADUs}^{-1}\right) \\
(7)\end{array}$ & $\begin{array}{c}\sigma_{\text {ref }} \\
\left(\mathrm{ADUs}^{-1}\right) \\
(8)\end{array}$ & $\begin{array}{c}f_{\text {diff }} \\
\left(\mathrm{ADUs}^{-1}\right) \\
(9)\end{array}$ & $\begin{array}{c}\sigma_{\text {diff }} \\
\left(\mathrm{ADUs}^{-1}\right) \\
(10)\end{array}$ & $\begin{array}{c}p \\
(11) \\
\end{array}$ \\
\hline V112 & I & 2456435.88824 & 13.706 & 4.679 & 0.001 & 141855.480 & 1550.259 & -44401.153 & 540.539 & 5.9879 \\
\hline V112 & I & 2456436.89695 & 13.689 & 4.661 & 0.001 & 141855.480 & 1550.259 & -30447.356 & 609.851 & 5.8368 \\
\hline$\vdots$ & $\vdots$ & $\vdots$ & $\vdots$ & $\vdots$ & $\vdots$ & $\vdots$ & $:$ & $:$ & $\vdots$ & $\vdots$ \\
\hline V160 & I & 2456435.88824 & 17.882 & 8.855 & 0.012 & 4069.406 & 1553.328 & -7171.335 & 186.330 & 5.9879 \\
\hline V160 & I & 2456436.89695 & 17.646 & 8.618 & 0.015 & 4069.406 & 1553.328 & -2916.178 & 292.135 & 5.8368 \\
\hline$\vdots$ & $\vdots$ & $\vdots$ & $\vdots$ & $\vdots$ & $\vdots$ & $\vdots$ & $\vdots$ & : & $\vdots$ & $\vdots$ \\
\hline V173 & I & 2456435.88824 & 17.745 & 8.717 & 0.011 & 3251.773 & 1764.129 & +39.643 & 192.483 & 5.9879 \\
\hline V173 & I & 2456436.89695 & 17.770 & 8.743 & 0.017 & 3251.773 & 1764.129 & -400.238 & 286.082 & 5.8368 \\
\hline$\vdots$ & $\vdots$ & $\vdots$ & $\vdots$ & $\vdots$ & $\vdots$ & $\vdots$ & $\vdots$ & $\vdots$ & $\vdots$ & $\vdots$ \\
\hline
\end{tabular}

Notes. The standard $M_{\text {std }}$ and instrumental $m_{\text {ins }}$ magnitudes are listed in Cols. 4 and 5, respectively, corresponding to the variable star, filter, and epoch of mid-exposure listed in Cols. 1-3, respectively. The uncertainty on $m_{\text {ins }}$ is listed in Col. 6, which also corresponds to the uncertainty on $M_{\text {std }}$. For completeness, we also list the quantities $f_{\text {ref }}, f_{\text {diff }}$, and $p$ from Eq. (2) in Cols. 7, 9, and 11, along with the uncertainties $\sigma_{\text {ref }}$ and $\sigma_{\text {diff }}$ in Cols. 8 and 10. This is an extract from the full table, which is available at the CDS.

To extract the photometry in each of the stacked images, we used the DanDIA ${ }^{2}$ pipeline (Bramich 2008; Bramich et al. 2013), which is based on difference image analysis (DIA; Alard \& Lupton 1998; Alard 2000). The pipeline works by aligning all images to the reference image, solving for a set of convolution kernels that were modelled as discrete pixel arrays, and subtracting the convolved reference image in each case to create a set of difference images. Stars are detected on the reference image and their reference fluxes $f_{\text {ref }}(\mathrm{ADU} / \mathrm{s})$ are measured using PSF photometry. Difference fluxes $f_{\text {diff }}(t)(\mathrm{ADU} / \mathrm{s})$ for each star detected in the reference image are measured in each of the difference images by optimally scaling the PSF model for the star to the difference image. The light curve for each star in instrumental magnitudes $m_{\text {ins }}$ are built as shown in Eq. (1):

$m_{\text {ins }}(t)=17.5-2.5 \log \left(f_{\text {tot }}(t)\right)$,

where $f_{\text {tot }}(t)$ is the total flux in ADU/s defined as

$f_{\mathrm{tot}}(t)=f_{\mathrm{ref}}+\frac{f_{\mathrm{diff}}(t)}{p(t)}$.

The quantity $p(t)$ is the photometric scale factor used to scale the reference frame to each image, as part of the kernel model explained in Bramich (2008). An electronic table with photometric measurements and fluxes for all the variable stars presented in this work is available through the $\mathrm{CDS}^{3}$ database with the format illustrated in Table 1.

\subsection{Astrometry and a finding chart}

To create a reference image with the astrometric information for each star in the field covered in NGC 6715, we used the celestial coordinates available in the ACS Globular Cluster Survey ${ }^{4}$ (see Anderson et al. 2008), which were uploaded for the field of the cluster through GAIA (Graphical Astronomy and Image Analysis Tool; Draper 2000). An $(x, y)$ shift to match their respective stars in our reference image was applied. Stars lying outside the

\footnotetext{
2 DanDIA is built from the DanIDL library of IDL routines available at http://www. danidl.co.uk

3 http://cds.u-strasbg.fr/

4 http://www.astro.ufl.edu/ ata/public_hstgc/
}

field of view and those without a clear match were removed and the $(x, y)$ shift was refined by minimising the squared coordinate residuals. A total of 305 stars over the entire field was used to guarantee that the astrometric solution applied to the reference image considered enough stars. The radial root mean square (rms) scatter obtained in the residuals was $\sim 00^{\prime \prime}$.028 ( $\sim 0.3$ pixels). This astrometrically calibrated reference image was used to produce a finding chart for NGC 6715 on which we marked the positions and identifications of all variable stars studied in this work (Fig. 5). Finally, a table with the equatorial J2000 celestial coordinates of all variables is given in Table A.1.

\section{Photometric calibration}

The photometric transformation of instrumental magnitudes to the standard system was accomplished using information available in the ACS Globular Cluster Survey, which provides calibrated magnitudes for selected stars in the fields of 50 globular clusters extracted from images taken with the Hubble Space Telescope (HST) instruments ACS and WFPC.

By matching the positions of the stars in the field of the HST images with those in our reference image, we obtained the photometric transformation shown in Fig. 2. The I magnitude obtained from the ACS (see Siegel et al. 2007) is plotted versus the instrumental $i^{\prime}+z^{\prime}$ magnitude obtained in this study. The red line is a linear fit with slope unity yielding the zero point labelled in the title, where $N$ is the number of stars used in the fit and $R$ is the correlation coefficient obtained. Owing to the substantial differences between the $i^{\prime}+z^{\prime}$ and standard I wavebands, there are non-linear colour terms in the transformation that we have not accounted for. However, we have opted for an approximate absolute photometric calibration since variable star discovery and classification do not require a precise calibration. Furthermore, our non-standard $i^{\prime}+z^{\prime}$ waveband precludes the possibility of using our RR Lyrae light curves for physical parameter estimation.

\section{Variable star searches}

Light curves for a total of 1405 stars were obtained with the DanDIA pipeline in the field covered by the reference image. To detect and extract the variable stars from all the non-variables, 


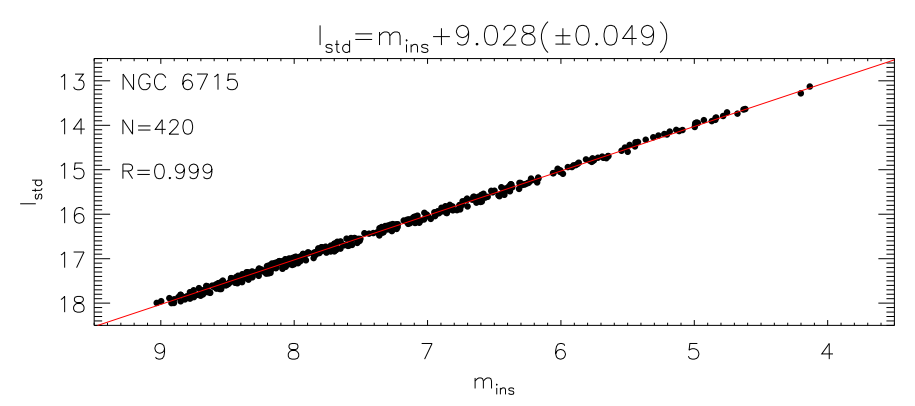

Fig. 2. Standard $I$ magnitude taken from the HST observations as a function of the instrumental $i^{\prime}+z^{\prime}$ magnitude. The red line is the fit that best matches the data and it is described by the equation in the title. The correlation coefficient is 0.999 .

three automatic (or semi-automatic) techniques were employed. They are described in Sects. 4.1-4.3.

\subsection{Root mean square}

A diagram of root mean square (rms) magnitude deviation against mean I magnitude (see top Fig. 3) was constructed for the cluster. In this diagram, we measure not only the photometric scatter for each star, but also the intrinsic variation of the variable stars over time, which gives them a higher rms than the non-variables. The classification is indicated by the colour as detailed in Table 2. To select candidate variable stars, we fit a polynomial to the rms values as a function of magnitude and flag all stars with an rms greater than two times the model value.

\section{2. $S_{B}$ statistic}

A detailed discussion can be found about the benefits of using the $S_{B}$ statistic to detect variable stars (Figuera Jaimes et al. 2013) and RR Lyrae with Blazhko effect (Arellano Ferro et al. 2012). The $S_{B}$ statistic is defined as

$S_{B}=\left(\frac{1}{N M}\right) \sum_{i=1}^{M}\left(\frac{r_{i, 1}}{\sigma_{i, 1}}+\frac{r_{i, 2}}{\sigma_{i, 2}}+\ldots+\frac{r_{i, k_{i}}}{\sigma_{i, k_{i}}}\right)^{2}$,

where $N$ is the number of data points for a given light curve and $M$ is the number of groups formed of time-consecutive residuals of the same sign from a constant-brightness light curve model (e. g. "mean or median"). The residuals $r_{i, 1}$ to $r_{i, k_{i}}$ correspond to the $i$ th group of $k_{i}$ time-consecutive residuals of the same sign with corresponding uncertainties $\sigma_{i, 1}$ to $\sigma_{i, k_{i}}$. The $S_{B}$ statistic is larger in value for light curves with long runs of consecutive data points above or below the mean, which is the case for variable stars with periods longer than the typical photometric cadence.

A plot of $S_{B}$ versus mean I magnitude is given for NGC 6715 (see bottom Fig. 3), variable stars are plotted in colour. To select candidate variable stars, the same technique employed in Sect. 4.1 was used, with the threshold also set at two times the model $S_{B}$ values.

\subsection{Stacked difference image}

Based on the results obtained using the DanDIA pipeline, a stacked difference image was built for NGC 6715 with the aim of detecting the difference fluxes that correspond to variable stars in the field of the reference image. The stacked image is the result of summing the absolute values of the difference images divided

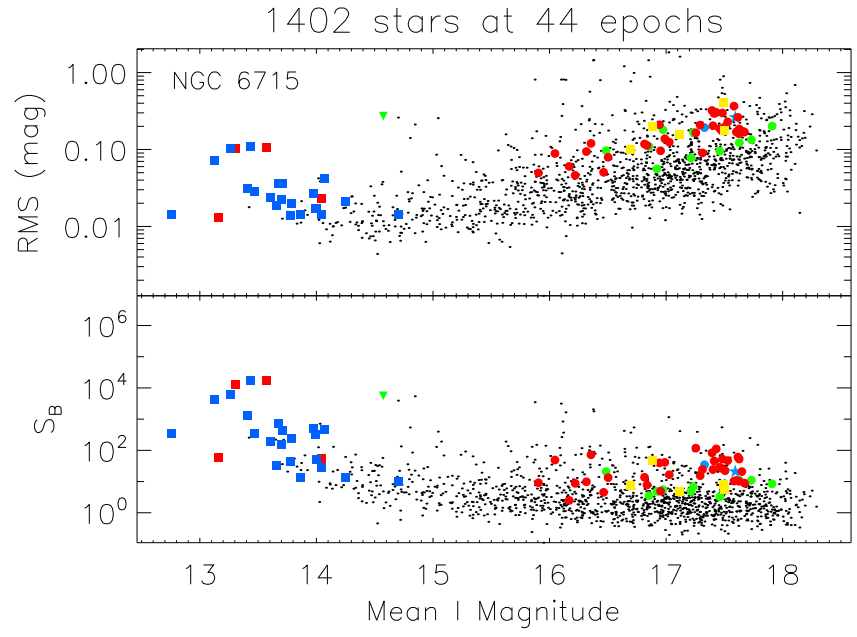

Fig. 3. Root mean square (rms) magnitude deviation (top) and $S_{B}$ statistic (bottom) versus the mean I magnitude for the 1402 stars detected in the field of view of the reference image for NGC 6715. Coloured points follow the convention adopted in Table 2 to identify the types of variables found in the field of this globular cluster.

Table 2. Convention used in the variable star classification of this work based on the definitions of the General Catalogue of Variable Stars (Samus et al. 2009).

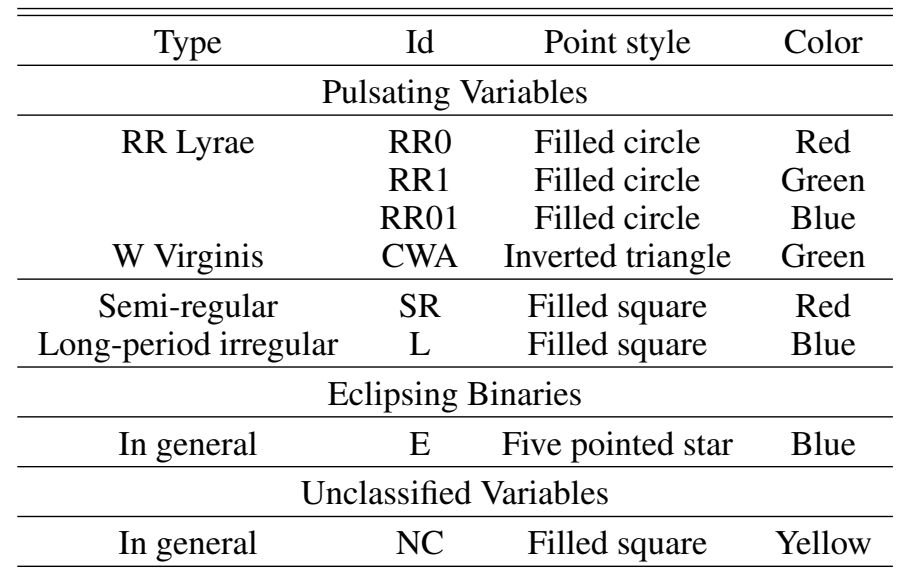

by the respective pixel uncertainty

$S_{i j}=\sum_{k} \frac{\left|D_{k i j}\right|}{\sigma_{k i j}}$

where $S_{i j}$ is the stacked image, $D_{k i j}$ is the $k$ th difference image, $\sigma_{k i j}$ is the pixel uncertainty associated with each image $k$, and the indexes $i$ and $j$ correspond to pixel positions.

All of the variable star candidates obtained by using the rms and $S_{B}$ diagrams explained in Sects. 4.1 and 4.2 were inspected visually in the stacked image and by blinking the difference images to confirm or refute their variability.

\section{Variable star classification}

To define the type of variation in each of the variable stars found, several steps were done. First, we used their position in the colour-magnitude diagram (CMD; Fig. 4) as a reference for their evolutionary stage as most types of variable stars are placed in very well defined zones in the CMD. Second, we implemented a period search for each of the light curves by using the string 


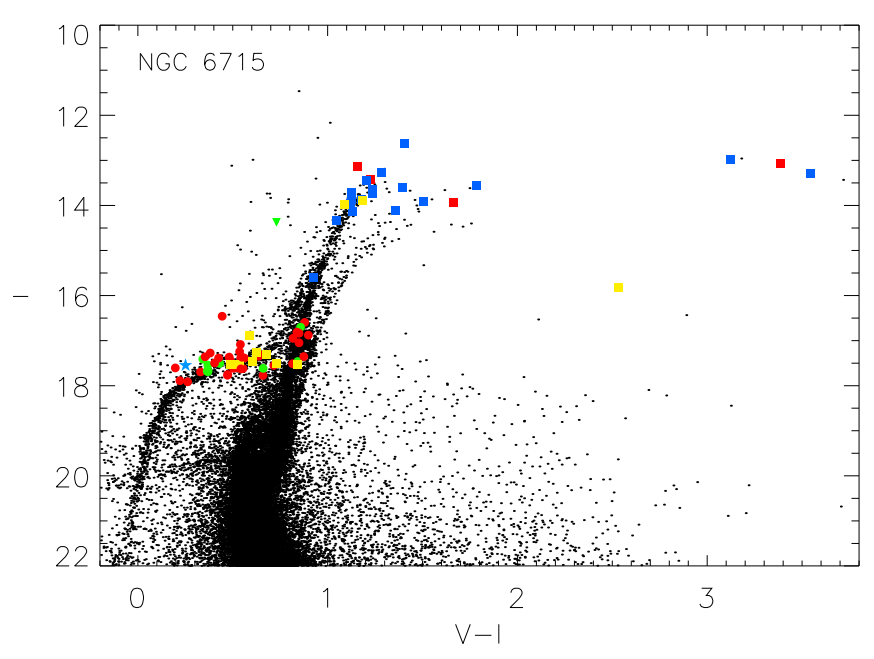

Fig. 4. Colour magnitude diagram of the globular cluster NGC 6715 built with $V$ and $I$ magnitudes available in the ACS globular cluster survey extracted from HST images.

method (Lafler \& Kinman 1965) and by minimising the $\chi^{2}$ in a Fourier analysis fit. Periods found and light curve shapes were also taken into account. Finally, to classify the variable stars, we used the conventions defined in the General Catalogue of Variable Stars (Samus et al. 2009).

In Table 2, the classification, corresponding symbols, and colours used in the plots throughout the paper are shown.

\section{Colour magnitude diagram}

Since our sample only has data available for one filter, we decided to build the colour-magnitude diagram (CMD; see Fig. 4) by using the information available from the HST images at the ACS Globular Cluster Survey. The data used correspond to the $V$ and $I$ photometry obtained in Sirianni et al. (2005). The CMD was useful in classifying the variable stars, especially those with poorly defined light curves such as long period variables and semi-regular variables, as well as corroborating cluster membership.

\section{NGC 6715/C1851-305/Messier 54}

The details of all variable stars in our FoV that are discussed in this section are listed in Table A.1, and all light curves are plotted in Fig. A.1.

\subsection{Known variables}

This globular cluster has of the order of 200 known variable stars listed in the Catalogue of Variable Stars in Galactic Globular Clusters (CVSGGC; Clement et al. 2001). Most of them are of the RR Lyrae type but a few are irregular (long-period type), semi-regular, W Virginis, eclipsing binaries, and SX Phoenicis. To date, four studies report variable star discoveries in this globular cluster: V1-V28 from Rosino (1952), V29-V82 from Rosino \& Nobili (1958), V83-V117 from Layden \& Sarajedini (2000), and V118-V211 from Sollima et al. (2010).

In the field of view covered by our reference image there are only five known variable stars (V112, V160, V173, V181, V192). All of them lie towards the edges of the image, as can be seen in Fig. 5. The star V112 was previously classified as irregular. However, we were able to find a period of $\sim 100$ days in the variability of this star and, because of this, we reclassified it as a semi-regular variable. For V160, we were not able to produce a good phased light curve using the published period of $0.6194848 \mathrm{~d}$. The discovery observations by Sollima et al. (2010) cover a time baseline of only six days. With our time baseline of more than two years, our derived periods are much more precise and the period found is in agreement with that found by the Optical Gravitational Lensing Experiment (OGLE, Udalski et al. 1992; Soszyński et al. 2014, see below). For V160, we list the OGLE period of $0.62813716 \mathrm{~d}$ in Table A.1. For V173, we improved the period estimate over that from Sollima et al. (2010). For V181, we find a very different period with respect to the one estimated by Sollima et al. (2010). The new period of $0.877072 \mathrm{~d}$ makes this RR Lyrae the one with the longest period in the cluster. The phased light curve in Fig. A.1 is somewhat noisy because this variable is highly blended with a brighter star. The case of V192 is particularly interesting because the star was classified as RR1 with a period of $0.3986799 \mathrm{~d}$. However, at the astrometric position reported for this star we found a RR Lyrae type RR0 with a period of $0.600373 \mathrm{~d}$. This is also in agreement with the period and classification found by OGLE (see below), which we list in Table A.1. Again, the phased light curve of this variable is noisy because of blending with a brighter star. It is clear then that the Sollima et al. (2010) periods and RR Lyrae classifications are not robust based on relatively few observations. We discuss the consequences of this later on.

Recently, Montiel \& Mighell (2010) announced 50 RR Lyrae candidates based on observations taken with the HST. However, the data obtained consist of 12 epochs covering only 8 hours, which made the study unsuitable for a period search and certainly some RR Lyrae stars will have been missed with such a short time baseline. No light curves were presented in their paper. Of these 50 candidate variable stars, 17 lie outside of our field of view (VC2, VC11-VC18, VC22, VC24, VC38, VC44, VC45, VC47-VC49). As pointed out in the Catalogue of Variable Stars in Galactic Globular Clusters (Clement et al. 2001), 11 of these candidates are previously known variables, thus $\mathrm{VC} 2=\mathrm{V} 127, \mathrm{VC} 11=\mathrm{V} 162, \mathrm{VC} 12=\mathrm{V} 163, \mathrm{VC} 13=\mathrm{V} 95$, $\mathrm{VC} 14=\mathrm{V} 164, \mathrm{VC} 15=\mathrm{V} 142, \mathrm{VC} 17=\mathrm{V} 129, \mathrm{VC} 18=\mathrm{V} 179$, VC44 = V46, VC45 = V148, and VC47 = V76 (see also Appendix B). For eight of their candidates in our field of view, we could not detect variations in our difference images at their coordinates (VC3, VC6, VC19, VC21, VC29, VC42, VC43, VC50), and we therefore cannot confirm their variability. We plot their positions in Fig. 5 with a green square ${ }^{5}$. Three of their candidates in our field of view, VC28, VC34, and VC46, are the known variables V181, V160, and V192, respectively. We confirm the variable nature of the remaining 22 candidates in our field of view, and we have assigned them " $V$ " numbers as part of our study (see Sect. 7.2). Table A.1 lists their VC identification in Col. 2. We classify 18 of them as RR Lyrae stars, one as an eclipsing binary, and are unable to classify VC27, VC32, and VC33. We plot their positions in Fig. 5 with a square symbol.

The study by McDonald et al. (2014) using the VISTA survey also covered the cluster and presents candidate variables based on typically $\sim 12-13$ epochs spread over $\sim 100-200$ days, which was insufficient to derive periods for many of them. Of the short period candidate variables listed in their Table 1, 18 are inside the field of view covered by our reference image. None of the bright irregular variables listed in their Table 2 and

5 The Montiel \& Mighell (2010) coordinates differ by RA $\sim 0.3^{\prime \prime}$ and Dec $\sim 0.8^{\prime \prime}$ from our coordinates and we have corrected for this in Fig. 5. 


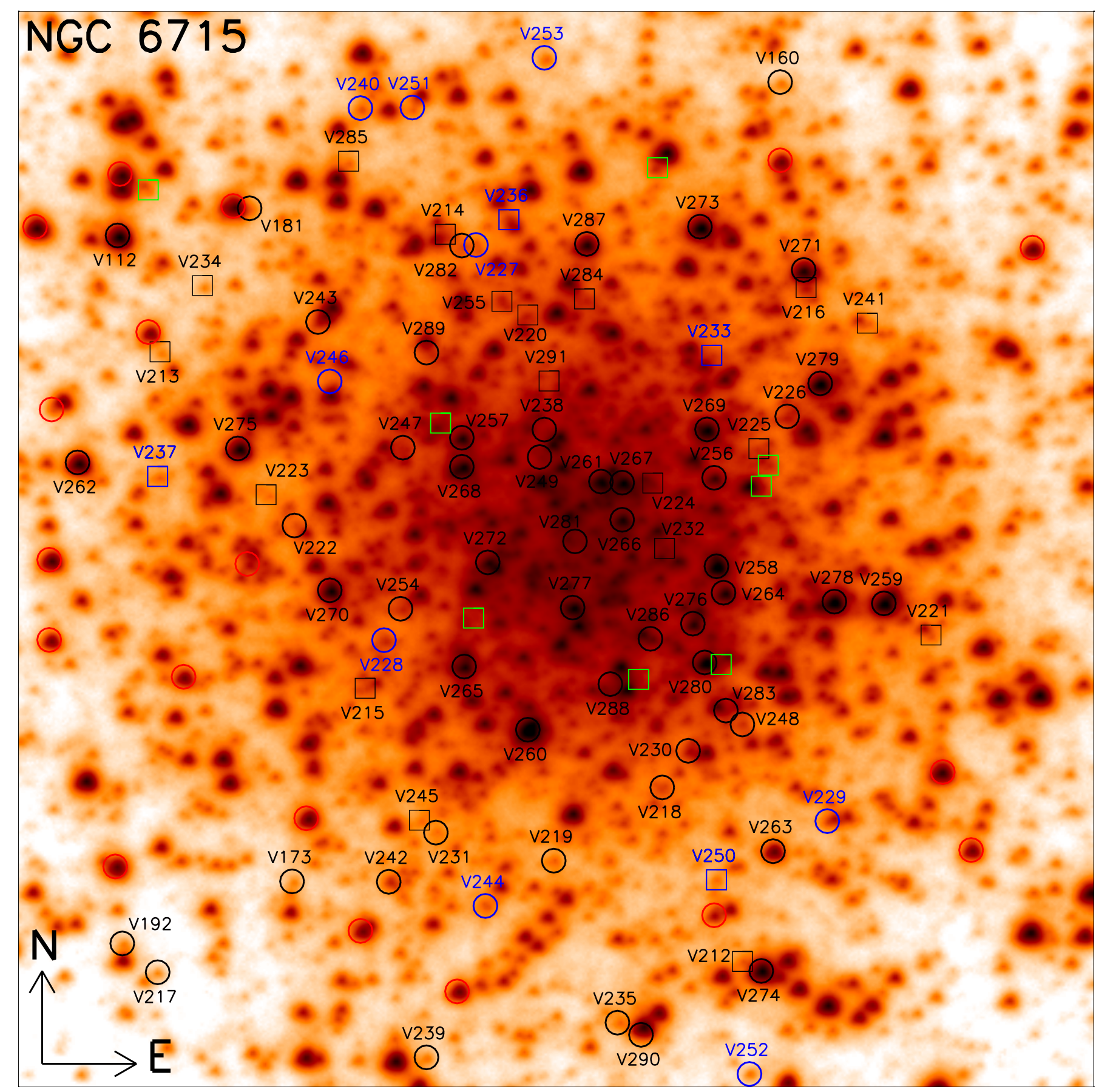

Fig. 5. Finding chart for the globular cluster NGC 6715. The image used corresponds to the reference image constructed during the reduction. All known variables and new discoveries are labelled with their V numbers. Known variables, or new variables discovered in this work, are plotted with black symbols. Variables discovered by the OGLE survey are plotted with blue symbols. Those variables that were candidate variables from Montiel \& Mighell (2010) are plotted using squares. Otherwise symbols are circles. Green squares and red circles, both without labels, are candidate variables from Montiel \& Mighell (2010) and McDonald et al. (2014), respectively, for which we do not detect variability in our survey. Image size is $\sim 40 \times 40 \operatorname{arcsec}^{2}$.

faint candidates listed in their Table 3 are inside the field covered in our study. Positions of these stars inside our field of view are plotted in Fig. 5 with a red circle. It is worth noting that all of these candidates are located more toward the edges of the reference image. We detect variability in only two of the McDonald et al. (2014) candidates within our field of view (SPVSgr18550405-3028580 and SPVSgr185503863028593, which we assign $V$ identifications as V229 and V263, respectively, see Sect. 7.2).
Similarly, the field of this cluster was also covered by OGLE, particularly with their OGLE-IV survey (Soszyński et al. 2014; Udalski et al. 2015). In this survey, we find 15 of the variable stars studied in our work, of which two are previously known variables (V160 and V192), and 13 are new discoveries by OGLE (we assigned the following $V$ identifications: V227-V229, V233, V236, V237, V240, V244, V246, V250V253). The OGLE light curves for these stars typically have $\sim 150$ epochs covering a baseline of $\sim 2.5$ yrs and OGLE derived 
precise periods for them. With our data, we were also able to find the same periods and type of classification assigned by OGLE. The positions of these stars are plotted in Fig. 5 with a blue colour. Again, it is worth noting that all of these variables are located more toward the edges of the reference image. In the particular case of V229, V244, and V246, the pipeline was not able to detect these stars in the reference image but their variation is clear in the difference images. Their differential fluxes against phase are plotted in Fig. A.1. Finally, epochs, periods, mean magnitudes, amplitudes, and classifications for these three stars were taken from the OGLE database, although the number of data points listed in Table A.1 correspond to our light curves.

In Appendix B (Table B.1), we provide the crossidentifications for the previously known RR Lyrae stars in NGC 6715 between the CVSGGC (Clement et al. 2001), the variable star candidates from Montiel \& Mighell (2010), and the OGLE RR Lyrae stars (Soszyński et al. 2014).

\subsection{New variables}

After employing the methods described in Sect. 4, we were able to extract 67 new variable stars in the core of NGC 6715 of which 30 are RR Lyrae, one is a W Virginis star (CWA), 21 are irregular (long-period type), three are semi regular, one is an eclipsing binary, and 11 remain without classification.

\subsubsection{RR Lyrae}

V213-V226, V230-V232, V234-V235, V238-V239, V241-V243, $V 245, V 247-V 249, V 254-V 255$ : these 30 newly discovered variable stars are clear RR Lyrae variables. Their positions in the CMD, light curve shapes, periods, and amplitudes corroborate their variability type. We found that 17 are pulsating in the fundamental mode (RR0); eight are pulsating in the first overtone (RR1); one is a double-mode pulsator (RR01) and four RR Lyrae remain with an uncertain subtype (3 RR0? and 1 RR1?). As shown in Table A.1, their periods range from $\sim 0.28 \mathrm{~d}$ to $\sim 0.76 \mathrm{~d}$, with amplitudes between 0.06 and 1.69 mag.

The periodogram analysis for V221 showed two predominant frequencies typical of double mode RR Lyrae stars, one equivalent to the fundamental period $P_{0}=0.459608 \mathrm{~d}$, and one equivalent to the first overtone period $P_{1}=0.343828 \mathrm{~d}$ giving a period ratio $P_{1} / P_{0}=0.748$, which falls into the expected ratio range of $\sim 0.725$ to $\sim 0.748$ for this type of pulsating RR Lyrae stars (Netzel et al. 2015; Moskalik 2013; Cox et al. 1983). Further data for a more detailed analysis of this star will be useful to corroborate its pulsational properties.

\subsubsection{W Virginis}

V256: particularly interesting is the case of this star, since its variation (and position in the CMD) do not follow the pattern found for the other variable stars studied and classified in this work. We found a very well phased light curve with a period of $\sim 14.771 \mathrm{~d}$ and an amplitude of $0.71 \mathrm{mag}$. This is the only bright variable star on the blue side of the colour-magnitude diagram far away from the red giant branch.

The properties found in the variation of this star and its position in the CMD match very well with the W Virginis-type of variable star described in Samus et al. (2009), particularly with the subtype CWA, which has periods longer than eight days (see also Wallerstein 2002). Although these types of stars have not been commonly found in globular clusters in contrast to RR Lyrae stars, they are not entirely uncommon. In the statistics of variable stars in Galactic globular clusters reported by Clement et al. (2001), it is possible to notice that 60 variable stars are Cepheids, which include Population II Cepheids, anomalous Cepheids, and RV Tauri stars. V256 is the first CWA star discovered in this cluster.

\subsubsection{Irregular}

V260-V280: these 21 stars are located at the top of the red giant branch, as shown with blue squares in Fig. 4. Their amplitudes range from 0.05 to $0.46 \mathrm{mag}$. We found no clear periods for these stars. Owing to this and also their position in the colour-magnitude diagram, we classified them as irregular. Light curves for all these variables are found in Fig. A.1.

\subsubsection{Semi regular}

V257-V259: based on the position of these three stars in the colour-magnitude diagram (see Fig. 4), their periods, and the shape of their light curves, we have classified them as semiregular. Light curves for these variables may be found in Fig. A.1. Their amplitudes range from $\sim 0.04$ to $\sim 0.45 \mathrm{mag}$ and their periods span between $\sim 20$ and $\sim 150 \mathrm{~d}$.

\subsubsection{Eclipsing binary}

V212: the light curve variations for this star are very similar to those presented in eclipsing binary systems. We found that the amplitude of the deeper eclipse is of the order of $\sim 0.8 \mathrm{mag}$ and the amplitude of the secondary eclipse is $\sim 0.5 \mathrm{mag}$. The phased light curve shown in Fig. A.1 represented a period of $P=0.202144 \mathrm{~d}$.

\subsubsection{Other variable stars}

V281-V285: these five stars are clear variable stars. They show clear variability by blinking the difference images. They have amplitudes between 0.45 and $0.94 \mathrm{mag}$. Several attempts to determine periods for these stars were done without success. As a result, their light curves in Fig. A.1 are plotted against HJD. We note that the variable source V281 is only $\sim 0.24$ arcsec from the photometric centre of the cluster, as measured by Goldsbury et al. (2010).

V286: towards the beginning of the 2013 data, we found that the flux of this star was increasing to a maximum at $\sim 2456464.9081 \mathrm{~d}$ of about $\sim 39400 \mathrm{ADU} / \mathrm{s}$ on the flux scale of the reference image, corresponding to a peak magnitude of $\sim 15.04$ mag. After that, its flux strongly decreased during the rest of the observational campaign. During 2014 and 2015, we found that the object seems to be at baseline and it is not detected in the original images.

The centre of NGC 6715 is $\sim 4.45$ arcseconds from this source. Also, at a distance of 3.73 arcseconds, there is an X-ray source studied by Wrobel et al. (2011) using data from Chandra and Hubble Space Telescope. Given the relatively small astrometric uncertainties in these positions, V286 is not associated with either. The nature and classification of this variable will need further studies, and it will remain without classification in this work.

V287-V291: these five stars were not detected by the pipeline in the reference image. Unfortunately, there are no light curves available in the OGLE database for these stars, but their 
variations are clear in the difference images. In Fig. A.1 their differential fluxes against HJD are plotted. As we were not able to produce phased light curves for them, they will remain without classification until future studies are done.

\section{Oosterhoff dichotomy}

In this work, we discovered 30 new RR Lyrae, and OGLE also discovered 17 more (of which 13 are inside our FoV). After removing the stars without a secure classification, there are 33 new RR0 and nine new RR1 stars, which represent a significant increment in the known RR Lyrae population. The vast majority of these new RR Lyrae stars are cluster members since we have studied the core of NGC 6715. Hence it is pertinent to recalculate the mean periods and number ratios of the RR Lyrae stars to see if they modify the current conclusions about the Oosterhoff type of NGC 6715.

However, we note that NGC 6715 lies projected against the Sagittarius dwarf spheroidal galaxy and behind the Galactic bulge. Therefore, the sample of RR Lyrae stars in the field of the cluster is a mixture of cluster members, bulge stars, and Sagittarius dwarf spheroidal (Sgr dSph) stars. Towards the centre of NGC 6715, the cluster member RR Lyraes dominate, but limiting our sample to the cluster core also reduces the number of RR Lyraes that can be used to calculate the mean periods and number ratios. Hence, in Fig. 6, we have plotted the total number of RR Lyrae stars, the mean period of the RR0 stars, the mean period of the RR1 stars, and the number ratio of the RR1 to all RR Lyrae stars (nRR1/(nRR0 + nRR1)), all as a function of distance from the cluster centre $(\mathrm{RA}(\mathrm{J} 2000)=$ 18:55:03.33; $\operatorname{Dec}(J 2000)=-30: 28: 47.5 ;$ Goldsbury et al. 2010). To convert angles on the sky into distances in parsecs, we used the distance from the Sun to NGC 6715 of 26500 pc (Harris 1996, 2010 version). The inner and outer red vertical lines are the tidal radii estimated by McLaughlin \& van der Marel (2005) based on King (1966) and Wilson (1975) models, respectively. The horizontal black lines correspond to the OoI (solid) and OoII (dashed) types given by (Smith 1995) in his Table 3.2. We only used RR Lyrae stars with certain classifications (i.e. published phased light curves with reliable period estimates).

Figure 6 clearly shows that within the range of the two estimates of the tidal radii, where the RR Lyrae stars from the cluster still dominate, the values of $\left\langle P_{\mathrm{RR} 0}\right\rangle,\left\langle P_{\mathrm{RR} 1}\right\rangle$ and nRR1/(nRR0 + nRR1) are intermediate between those expected for OoI and OoII clusters. We obtain $\left\langle P_{\mathrm{RR} 0}\right\rangle=0.613529 \mathrm{~d}$, $\left\langle P_{\mathrm{RR} 1}\right\rangle=0.334107 \mathrm{~d}$, and $\mathrm{nRR} 1 /(\mathrm{nRR} 0+\mathrm{nRR} 1)=0.24050633$ when calculated for the 79 RR Lyraes within the outer estimate of the tidal radius (i.e. $380 \mathrm{pc}$ ). The contaminating RR Lyrae populations have $\left(\left\langle P_{\mathrm{RR} 0}\right\rangle,\left\langle P_{\mathrm{RR} 1}\right\rangle\right)=(0.556 \mathrm{~d}, 0.310 \mathrm{~d})$ and $\left(\left\langle P_{\mathrm{RR} 0}\right\rangle,\left\langle P_{\mathrm{RR} 1}\right\rangle\right)=(0.574 \mathrm{~d}, 0.322 \mathrm{~d})$ for the bulge and Sgr dSph, respectively (Soszyński et al. 2011; Cseresnjes 2001), placing them in the OoI region in the $\left\langle P_{\mathrm{RR} 0}\right\rangle$-metallicity diagram of Catelan (2009). This explains why the values of $\left\langle P_{\mathrm{RR} 0}\right\rangle$ and $\mathrm{nRR} 1 /(\mathrm{nRR} 0+\mathrm{nRR} 1)$ tend towards the OoI values beyond the outer estimate of the tidal radius. The value of $\left\langle P_{\mathrm{RR} 1}\right\rangle$ is hardly influenced though, because there are only six RR1 stars beyond the outer estimate of the tidal radius. Hence the new RR Lyrae discoveries in this paper have served to confirm that NGC 6715 is of intermediate Oosterhoff type.

\section{Conclusions}

The globular cluster NGC 6715 turns out to be a very interesting stellar system, for which images with the highest angular

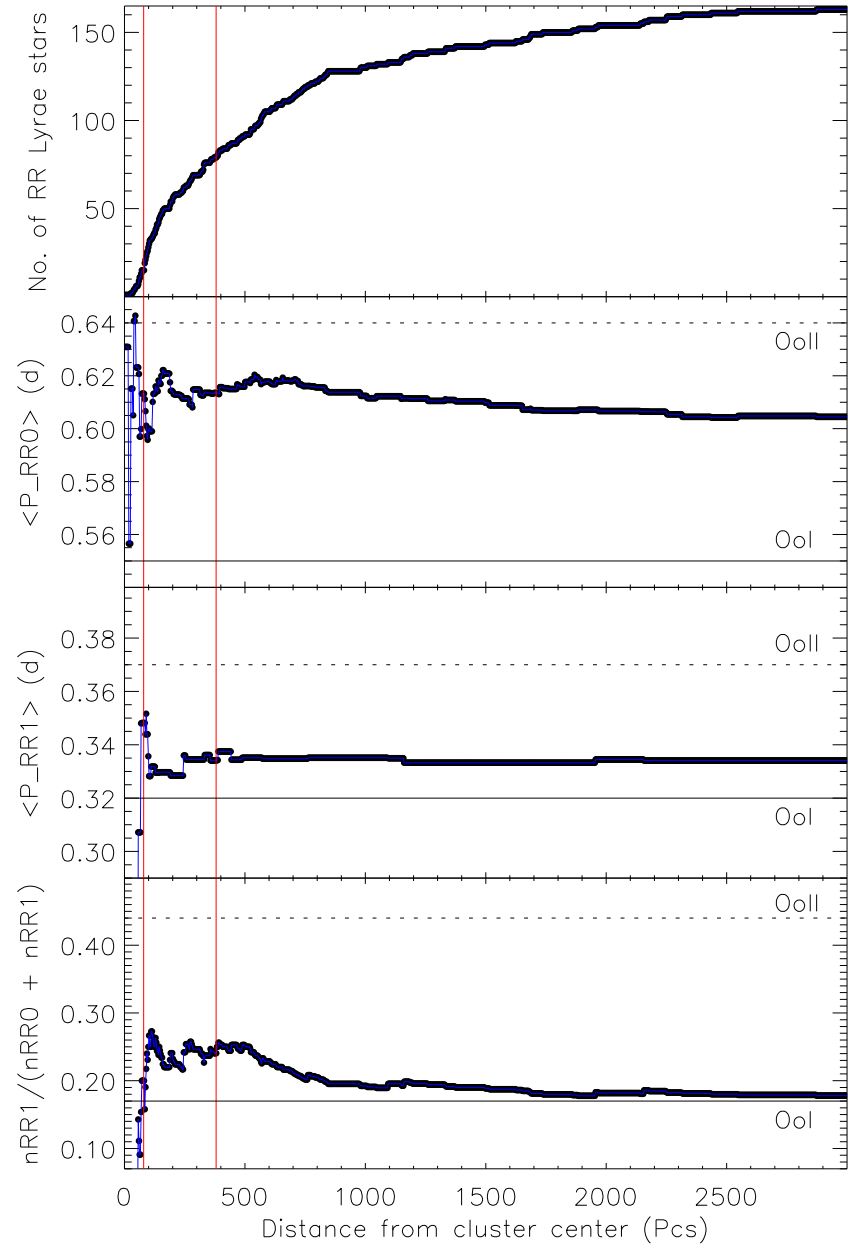

Fig. 6. The number of RR Lyraes, mean periods, and number ratios as a function of the distance from the cluster centre. The red lines correspond to the tidal radii calculated by McLaughlin \& van der Marel (2005).

resolution have ever been obtained so far with ground-based telescopes. The use of the EMCCD and shift-and-add technique was demonstrated to be an excellent procedure to minimise the effect of the atmospheric turbulence, which is one of the main constraints when doing ground-based observations. Thanks to this and the use of difference image analysis, it was possible to obtain high-precision time series photometry in the core of this cluster down to $I \sim 18.3 \mathrm{mag}$.

A total of 1405 stars in the field covered by the reference image were statistically studied for variable star detection. We presented light curves for 17 previously known variables that were found toward the edges of the reference image (16 RR Lyrae and $1 \mathrm{SR})$. We also discovered 67 new variable stars, which consist of 30 RR Lyrae, 21 irregular (long-period type), three semi-regular, $1 \mathrm{~W}$ Virginis, one eclipsing binary, and 11 unclassified stars. We estimated periods and ephemerides for all variable stars in the field of our reference image. Our new RR Lyrae star discoveries help confirm that NGC 6715 is of intermediate Oosterhoff type. Finally, our photometric measurements for all variable stars studied in this work are available in electronic form through the Strasbourg Astronomical Data Centre.

Acknowledgements. Our thanks go to Christine Clement for clarifying the known variable star content in NGC 6715 and the numbering systems of the variable stars while we were working on these clusters. This support to the astronomical community is very much appreciated. The Danish $1.54 \mathrm{~m}$ telescope is operated based on a grant from the Danish Natural Science Foundation (FNU). 
This publication was made possible by NPRP grant \# X-019-1-006 from the Qatar National Research Fund (a member of Qatar Foundation). The statements made herein are solely the responsibility of the authors. K.H. acknowledges support from STFC grant ST/M001296/1. G.D. acknowledges Regione Campania for support from POR-FSE Campania 2014-2020. D.F.E. is funded by the UK Science and Technology Facilities Council. T.H. is supported by a Sapere Aude Starting Grant from the Danish Council for Independent Research. Research at Centre for Star and Planet Formation is funded by the Danish National Research Foundation. T.C.H. acknowledges support from the Korea Research Council of Fundamental Science \& Technology (KRCF) via the KRCF Young Scientist Research Fellowship. Programme and for financial support from KASI travel grant number 2013-9-400-00, 2014-1-400-06 \& 2015-1-850-04. N.P. acknowledges funding by the Gemini-Conicyt Fund, allocated to project No. 32120036 and by the Portuguese FCT - Foundation for Science and Technology and the European Social Fund (ref: SFRH/BGCT/113686/2015). CITEUC is funded by National Funds through FCT - Foundation for Science and Technology (project: UID/Multi/00611/2013) and FEDER - European Regional Development Fund through COMPETE 2020 - Operational Programme Competitiveness and Internationalisation (project: POCI-01-0145-FEDER-006922). OW and J. Surdej acknowledge support from the Communauté française de Belgique - Actions de recherche concertées - Académie Wallonie-Europe. This work has made extensive use of the ADS and SIMBAD services, for which we are thankful.

Note added in proof. During the final stages of the refereeing process for this paper, Hamanowicz et al. (2016) posted a draft paper on the arXiv preprint website on the subject of variable stars in Sgr dSph and M54. They used OGLE imaging data with conventional CCDs, covering a much larger field of view than our work, although our images are of better resolution having been obtained with EMCCDs. While both sets of results are complementary, there are however a substantial number of variables that have been independently discovered by ourselves and Hamanowicz et al. (2016). These stars are indicated in Col. 2 of Table A.1 with the term O16. Both teams agreed to work together to form a consistent naming convention for the new variable stars with the aim of avoiding confusion for future researchers. We believe that the positive scientific spirit shown by both teams to solve this issue to the benefit of the astronomical community is commendable.

\section{References}

Alard, C. 2000, A\&AS, 144, 363

Alard, C., \& Lupton, R. H. 1998, ApJ, 503, 325

Anderson, J., Sarajedini, A., Bedin, L. R., et al. 2008, AJ, 135, 2055

Arellano Ferro, A., Bramich, D. M., Figuera Jaimes, R., Giridhar, S., \& Kuppuswamy, K. 2012, MNRAS, 420, 1333

Bellazzini, M., Ibata, R. A., Chapman, S. C., et al. 2008, AJ, 136, 1147

Bessell, M. S. 2005, ARA\&A, 43, 293

Bozza, V., Shvartzvald, Y., Udalski, A., et al. 2016, ApJ, 820, 79

Bramich, D. M. 2008, MNRAS, 386, L77

Bramich, D. M., Horne, K., Albrow, M. D., et al. 2013, MNRAS, 428, 2275

Carretta, E., Bragaglia, A., Gratton, R. G., et al. 2010, A\&A, 520, A95

Catelan, M. 2004, in IAU Colloq. 193: Variable Stars in the Local Group, eds. D. W. Kurtz, \& K. R. Pollard, ASP Conf. Ser., 310, 113

Catelan, M. 2009, Ap\&SS, 320, 261

Ciceri, S., Mancini, L., Southworth, J., et al. 2016, MNRAS, 456, 990

Clement, C. M., Muzzin, A., Dufton, Q., et al. 2001, AJ, 122, 2587

Cox, A. N., Hodson, S. W., \& Clancy, S. P. 1983, ApJ, 266, 94

Cseresnjes, P. 2001, A\&A, 375, 909

Draper, P. W. 2000, in Astronomical Data Analysis Software and Systems IX, eds. N. Manset, C. Veillet, \& D. Crabtree, ASP Conf. Ser., 216, 615

Evans, D. F., Southworth, J., Maxted, P. F. L., et al. 2016, A\&A, 589, A58

Figuera Jaimes, R., Arellano Ferro, A., Bramich, D. M., Giridhar, S., \& Kuppuswamy, K. 2013, A\&A, 556, A20

Figuera Jaimes, R., Bramich, D. M., Skottfelt, J., et al. 2016, A\&A, 588, A128 Goldsbury, R., Richer, H. B., Anderson, J., et al. 2010, AJ, 140, 1830

Hamanowicz, A., Pietrukowicz, P., Udalski, A., et al. 2016, Acta Astron., 66, 197

Harpsøe, K. B. W., Jørgensen, U. G., Andersen, M. I., \& Grundahl, F. 2012, A\&A, 542, A23

Harris, W. E. 1996, AJ, 112, 1487
Ibata, R., Bellazzini, M., Chapman, S. C., et al. 2009, ApJ, 699, L169

Jerram, P., Pool, P. J., Bell, R., et al. 2001, in Sensors and Camera Systems for Scientific, Industrial, and Digital Photography Applications II, eds. M. M. Blouke, J. Canosa, \& N. Sampat, SPIE Conf. Ser., 4306, 178

King, I. R. 1966, AJ, 71, 64

Kinman, T. D. 1959, MNRAS, 119, 134

Lafler, J., \& Kinman, T. D. 1965, ApJS, 11, 216

Layden, A. C., \& Sarajedini, A. 2000, AJ, 119, 1760

Lee, J.-W., \& Carney, B. W. 1999, AJ, 118, 1373

Li, K., \& Qian, S.-B. 2013, New Astron., 22, 57

Majewski, S. R., Skrutskie, M. F., Weinberg, M. D., \& Ostheimer, J. C. 2003, ApJ, 599, 1082

Mancini, L., Giacobbe, P., Littlefair, S. P., et al. 2015, A\&A, 584, A104

McDonald, I., Zijlstra, A. A., Sloan, G. C., et al. 2014, MNRAS, 439, 2618

McLaughlin, D. E., \& van der Marel, R. P. 2005, ApJS, 161, 304

Milone, A. P. 2015, ArXiv e-prints [arXiv: 1510.02578]

Monaco, L., Bellazzini, M., Ferraro, F. R., \& Pancino, E. 2005, MNRAS, 356, 1396

Montiel, E. J., \& Mighell, K. J. 2010, AJ, 140, 1500

Moskalik, P. 2013, in Stellar Pulsations: Impact of New Instrumentation and New Insights, eds. J. C. Suárez, R. Garrido, L. A. Balona, \& J. ChristensenDalsgaard, Astrophys. Space Sci. Proc., 31, 103

Netzel, H., Smolec, R., \& Dziembowski, W. 2015, MNRAS, 451, L25

Oosterhoff, P. T. 1939, The Observatory, 62, 104

Pryor, C., \& Meylan, G. 1993, in Structure and Dynamics of Globular Clusters, eds. S. G. Djorgovski, \& G. Meylan, ASP Conf. Ser., 50, 357

Rosenberg, A., Recio-Blanco, A., \& García-Marín, M. 2004, ApJ, 603, 135

Rosino, L. 1952, Mem. Soc. Astron. It. , 23, 49

Rosino, L., \& Nobili, F. 1958, Mem. Soc. Astron. It., 29, 413

Samus, N. N., Durlevich, O. V., \& et al. 2009, VizieR Online Data Catalog: II/25

Siegel, M. H., Dotter, A., Majewski, S. R., et al. 2007, ApJ, 667, L57

Sirianni, M., Jee, M. J., Benítez, N., et al. 2005, PASP, 117, 1049

Skottfelt, J., Bramich, D. M., Figuera Jaimes, R., et al. 2013, A\&A, 553, A111

Skottfelt, J., Bramich, D. M., Figuera Jaimes, R., et al. 2015a, A\&A, 573, A103

Skottfelt, J., Bramich, D. M., Hundertmark, M., et al. 2015b, A\&A, 574, A54

Smith, H. A. 1995, Cambridge Astrophys. Ser., 27

Smith, N., Giltinan, A., O'Connor, A., et al. 2008, in Astrophys. Space Sci. Libr. 351, eds. D. Phelan, O. Ryan, \& A. Shearer, 257

Smith, H. A., Catelan, M., \& Kuehn, C. 2011, in RR Lyrae Stars, Metal-Poor Stars, and the Galaxy, Carnegie Obs. Astrophys. Ser. 5, ed. A. McWilliam, 17

Sollima, A., Cacciari, C., Bellazzini, M., \& Colucci, S. 2010, MNRAS, 406, 329

Sollima, A., Cassisi, S., Fiorentino, G., \& Gratton, R. G. 2014, MNRAS, 444, 1862

Soszyński, I., Udalski, A., Pietrukowicz, P., et al. 2011, Acta Astron., 61, 285

Soszyński, I., Udalski, A., Szymański, M. K., et al. 2014, Acta Astron., 64, 177

Southworth, J., Mancini, L., Tregloan-Reed, J., et al. 2015, MNRAS, 454, 3094

Street, R. A., Udalski, A., Calchi Novati, S., et al. 2016, ApJ, 819, 93

Udalski, A., Szymanski, M., Kaluzny, J., Kubiak, M., \& Mateo, M. 1992, Acta Astron., 42, 253

Udalski, A., Szymański, M. K., \& Szymański, G. 2015, Acta Astron., 65, 1

Wallerstein, G. 2002, PASP, 114, 689

Wilson, C. P. 1975, AJ, 80, 175

Wrobel, J. M., Greene, J. E., \& Ho, L. C. 2011, AJ, 142, 113

${ }^{1}$ SUPA, School of Physics and Astronomy, University of St. Andrews, North Haugh, St Andrews, KY16 9SS, UK e-mail: robertofiguera@gmail.com

2 European Southern Observatory, Karl-Schwarzschild-Straße 2, 85748 Garching bei München, Germany

3 Qatar Environment and Energy Research Institute (QEERI), HBKU, Qatar Foundation, Doha, Qatar

${ }^{4}$ Centre for Electronic Imaging, Dept. of Physical Sciences, The Open University, Milton Keynes MK7 6AA, UK

5 Niels Bohr Institute and Centre for Star and Planet Formation, University of Copenhagen, Øster Voldgade 5, 1350 Copenhagen K, Denmark

6 Space Telescope Science Institute, 3700 San Martin Drive, Baltimore, MD 21218, USA

7 Dipartimento di Fisica "E. R. Caianiello", Università di Salerno, via Giovanni Paolo II 132, 84084 Fisciano, Italy

8 Istituto Nazionale di Fisica Nucleare, Sezione di Napoli, 80126 Napoli, Italy 
9 NASA Exoplanet Science Institute, MS 100-22, California Institute of Technology, Pasadena CA 91125, USA

10 Istituto Internazionale per gli Alti Studi Scientifici (IIASS), 84019 Vietri Sul Mare, Italy

11 Max Planck Institute for Astronomy, Königstuhl 17, 69117 Heidelberg, Germany

12 Yunnan Observatories, Chinese Academy of Sciences, 650011 Kunming, PR China

13 Key Laboratory for the Structure and Evolution of Celestial Objects, Chinese Academy of Sciences, 650011 Kunming, PR China

14 Korea Astronomy and Space Science Institute, Daejeon 305-348, Republic of Korea

15 Finnish Centre for Astronomy with ESO (FINCA), University of Turku, Väisäläntie 20, 21500 Piikkiö, Finland

16 Instituto de Astrofísica, Facultad de Física, Pontificia Universidad Católica de Chile, Av. Vicuña Mackenna 4860, 7820436 Macul, Santiago, Chile

17 Department of Physics, Sharif University of Technology, PO Box 11155-9161 Tehran, Iran

18 Astronomisches Rechen-Institut, Zentrum für Astronomie der Universität Heidelberg, Mönchhofstr. 12-14, 69120 Heidelberg, Germany

19 Planetary and Space Sciences, Department of Physical Sciences, The Open University, Milton Keynes, MK7 6AA, UK
20 Max-Planck-Institute for Solar System Research, Justus-vonLiebig-Weg 3, 37077 Göttingen, Germany

21 Astrophysics Group, Keele University, Staffordshire, ST5 5BG, UK

22 Las Cumbres Observatory Global Telescope Network, 6740 Cortona Drive, Suite 102, Goleta, CA 93117, USA

23 Institut d'Astrophysique et de Géophysique, Université de Liège, Allée du 6 Août $9 \mathrm{c}, 4000$ Liège, Belgium

24 Dark Cosmology Centre, Niels Bohr Institute, University of Copenhagen, Juliane Maries vej 30, 2100 Copenhagen $\varnothing$, Denmark

25 Department of Astronomy, Stockholm University, AlbaNova University Center, 10691 Stockholm, Sweden

26 Meteorologisches Institut, Universität Hamburg, Bundesstraße 55, 20146 Hamburg, Germany

27 Universidad de Antofagasta, Unidad de Astronomía, Facultad Cs. Básicas, Av. U. de Antofagasta, 02800 Antofagasta, Chile

2820 CITEUC - Centre for Earth and Space Science Research of the University of Coimbra, Observatório Astronómico da Universidade de Coimbra, 3040-004 Coimbra, Portugal

29 Jodrell Bank Centre for Astrophysics, School of Physics and Astronomy, University of Manchester, Oxford Road, Manchester M139PL, UK

30 Stellar Astrophysics Centre, Department of Physics and Astronomy, Aarhus University, Ny Munkegade 120, 8000 Aarhus C, Denmark 


\section{Appendix A: Additional tables and figures}

Table A.1. Ephemerides and main characteristics of the variable stars in the field of globular cluster NGC 6715.

\begin{tabular}{|c|c|c|c|c|c|c|c|c|c|}
\hline $\begin{array}{l}\text { Var } \\
\text { id } \\
(1)\end{array}$ & $\begin{array}{l}\text { Other } \\
\text { id } \\
(2)\end{array}$ & $\begin{array}{c}\text { RA } \\
\text { J2000 } \\
(3)\end{array}$ & $\begin{array}{c}\text { Dec } \\
\text { J2000 } \\
(4) \\
\end{array}$ & $\begin{array}{c}\text { Epoch } \\
\text { HJD } \\
(5)\end{array}$ & $\begin{array}{c}P \\
\mathrm{~d} \\
(6) \\
\end{array}$ & $\begin{array}{c}I_{\text {median }} \\
\text { mag } \\
(7)\end{array}$ & $\begin{array}{c}A_{i^{\prime}+z^{\prime}} \\
\operatorname{mag} \\
(8)\end{array}$ & (9) & (10) \\
\hline V112 & - & $18: 55: 02.010$ & $-30: 28: 35.13$ & 2457190.7419 & $100(1)$ & 13.63 & & & $\mathrm{SR}$ \\
\hline V160 & VC34; 37595 & $18: 55: 03.954$ & $-30: 28: 29.83$ & 2457189.7725 & $0.62813716(382)$ & 17.65 & & 40 & RR0 \\
\hline V173 & - & $18: 55: 02.473$ & $-30: 29: 00.04$ & 2457206.6555 & $0.360284(152)$ & 17.69 & 0.30 & 44 & RR1 \\
\hline V181 & VC28 & $18: 55: 02.395$ & $-30: 28: 34.20$ & 2457145.9107 & $0.877072(898)$ & 17.60 & 0.87 & 42 & RR0 \\
\hline V192 & VC46; 37568 & 18:55:01.973 & $-30: 29: 02.27$ & 2456784.9179 & $0.60035438(1060)$ & 17.63 & 0.60 & 44 & RR0 \\
\hline V212 & VC35 & $18: 55: 03.777$ & $-30: 29: 03.51$ & 2457214.8470 & & 17.59 & & 42 & $\mathrm{E}$ \\
\hline $\mathrm{V} 213$ & O16; VC40 & $18: 55: 02.126$ & $-30: 28: 39.63$ & 2456771.8623 & $0.286053(96)$ & 17.95 & 0.64 & 44 & RR1 \\
\hline V214 & O16; VC39 & $18: 55: 02.964$ & $-30: 28: 35.37$ & 2456765.9179 & $0.305386(109)$ & 16.97 & 0.45 & 44 & RR1 \\
\hline $\mathrm{V} 215$ & O16; VC25 & $18: 55: 02.701$ & $-30: 28: 52.71$ & 2456789.8857 & $0.307160(110)$ & 17.45 & 0.34 & 44 & RR1 \\
\hline V216 & VC9 & & $-30: 28: 3$ & 2457292 . & & 16.49 & .20 & 44 & RR1 \\
\hline V217 & $\mathrm{O} 16$ & $18: 55: 02.070$ & $-30: 29: 03.41$ & 2457205.7553 & & 17.76 & 0.35 & 44 & RR1 \\
\hline V218 & - & 18:55:03.556 & $-30: 28: 56.77$ & 2456891.5200 & $0.348450(142)$ & 16.92 & 0.20 & 44 & RR1 \\
\hline V219 & O16 & $18: 55: 03.233$ & $-30: 28: 59.48$ & 2457183.7770 & $0.382637(171)$ & 17.21 & 0.24 & 44 & RR1 \\
\hline V220 & O16; VC4 & 18:55:03.197 & $-30: 28: 38.54$ & 2456765. & 0.388893 & 16.86 & & 44 & RR1 \\
\hline V221 & VC8 & & $-30: 28: 51.16$ & & & 17.39 & & 44 & RR01 \\
\hline V222 & - & $18: 55: 02.504$ & $-30: 28: 46.40$ & & & 17.70 & 0.70 & 44 & RR0? \\
\hline V223 & O16; VC10 & $18: 55: 02.425$ & $-30: 28: 45.20$ & 2456823.8857 & $0.471901(260)$ & 17.63 & 0.81 & 44 & RR0 \\
\hline V224 & VC20 & $18: 55: 03.549$ & $-30: 28: 45.09$ & 2457292.6392 & $0.482336(272)$ & 16.16 & 0.27 & 44 & RR0? \\
\hline V225 & VC37 & 62 & $-30: 28: 4$ & 2456464 & & 17.28 & 0.35 & 44 & RR1? \\
\hline V226 & - & $18: 55: 03.948$ & $-30: 28: 42.65$ & 2456784.9179 & 0.497941 & 16.39 & 0.36 & 44 & RR0? \\
\hline V227 & 37582 & $18: 55: 03.050$ & $-30: 28: 35.81$ & 2457190.7991 & $0.50805795(4$ & 17.66 & 0.90 & 44 & RR0 \\
\hline V228 & 37575 & $18: 55: 02.757$ & $-30: 28: 50.88$ & 2456534.6709 & $0.52522562(844)$ & 16.87 & 0.41 & 44 & RR0 \\
\hline & $37597^{b}$ & & $-30: 28: 58.20$ & & & 16.38 & 0.28 & 41 & RR0 \\
\hline V230 & O16 & & & 2456465.8 & & 16.04 & 0.29 & 44 & RR0 \\
\hline V231 & O16 & $18: 55: 02.894$ & $-30: 28: 58.30$ & 2457189.8090 & $0.534194(333)$ & 17.80 & 0.83 & 44 & RR0 \\
\hline V232 & VC41 & $18: 55: 03.581$ & $-30: 28: 47.60$ & 2456543.5260 & & 16.35 & 0.29 & 44 & RR0 \\
\hline V233 & VC30; 37591 & & $-30: 28: 4$ & & & 17.41 & & 44 & RR0 \\
\hline V234 & O16; VC7 & $18: 55: 02.255$ & $-30: 28: 37.13$ & 2457206.6400 & $0.559765(366)$ & 17.72 & 0.60 & 44 & RR0 \\
\hline V235 & - & 18:55:03.411 & $-30: 29: 05.75$ & 2457292.6392 & $0.566711(375)$ & 17.41 & 0.69 & 43 & RR0 \\
\hline V236 & VC36; 37585 & 18:55:03.149 & $-30: 28: 34.86$ & 2457152.8500 & $0.56847125(715)$ & 16.53 & 0.28 & 44 & RR0 \\
\hline V237 & VC31; 37570 & & $-30: 28: 44.40$ & 2456435.8882 & & 17.57 & 65 & 44 & RR0 \\
\hline & - & & & & & & & 44 & RR0 \\
\hline V239 & 016 & $18: 55: 02.848$ & $-30: 29: 06.91$ & 2457189.8090 & $0.596179(432)$ & 17.57 & 0.69 & 39 & RR0 \\
\hline V240 & 37576 & $18: 55: 02.725$ & $-30: 28: 30.46$ & 2456909.5275 & $0.59629933(890)$ & 17.06 & 0.54 & 43 & RR0 \\
\hline V241 & O16; VC1 & $18: 55: 04.190$ & $-30: 28: 39.15$ & 2456896.5087 & $0.602588(424)$ & 17.79 & 0.65 & 44 & RR0 \\
\hline V242 & O16 & $18: 55: 02.756$ & $-30: 29: 00.15$ & 2456891.4700 & $0.604762(427)$ & 16.23 & 0.16 & 44 & RR0 \\
\hline V243 & - & $18: 55: 02.587$ & $-30: 28: 38.62$ & 2456770.8691 & $0.610170(435)$ & 16.97 & 0.36 & 44 & RR0 \\
\hline $\mathrm{V} 244^{a}$ & 37581 & $18: 55: 03.031$ & $-30: 29: 01.15$ & 2456891.4945 & $0.61517949(819)$ & 16.90 & 0.27 & 44 & RR0 \\
\hline V245 & VC26 & $18: 55: 02.849$ & $-30: 28: 57.81$ & 2456771.8623 & $0.626797(459)$ & 17.68 & 0.47 & 44 & RR0 \\
\hline $\mathrm{V} 246^{a}$ & 37573 & $18: 55: 02.616$ & $-30: 28: 40.91$ & 2457207.8691 & $0.62879467(894)$ & 16.11 & 0.06 & 42 & RR0 \\
\hline V247 & O16 & $18: 55: 02.826$ & $-30: 28: 43.51$ & 2456846.9179 & $0.650946(495)$ & 16.86 & 0.42 & 44 & RR0 \\
\hline V248 & - & 18:55:03.795 & $-30: 28: 54.42$ & 2457258.7147 & $0.668967(522)$ & 17.05 & 0.46 & 44 & RR0 \\
\hline V249 & - & $18: 55: 03.226$ & $-30: 28: 43.99$ & 2457191.7238 & $0.673703(530)$ & 15.91 & 0.21 & 44 & RR0 \\
\hline $\mathrm{V} 250$ & VC23; 37590 & $18: 55: 03.705$ & $-30: 29: 00.36$ & 2456543.5260 & $0.68064951(790)$ & 17.54 & 0.58 & 44 & RR0 \\
\hline V251 & 37579 & $18: 55: 02.876$ & $-30: 28: 30.48$ & 2457189.7725 & $0.68928616(716)$ & 17.54 & 0.88 & 43 & RR0 \\
\hline V252 & 37593 & 18:55:03.792 & $-30: 29: 07.83$ & 2457189.8090 & $0.72866844(702)$ & 17.28 & 0.50 & 17 & RR0 \\
\hline
\end{tabular}

Notes. Column 1 is the id assigned to the variable star; Col. 2 is a previously known id assigned to the stars (5 digit numbers correspond to OGLE identifications of the form OGLE-BLG-RRLYR-NNNNN; O16 indicates that the variable star was discovered independently by Hamanowicz et al. 2016, see note added in proof after our conclusions), Cols. 3 and 4 correspond to the right ascension and declination (J2000); Col. 5 is the epoch used; Col. 6 is the period measured in this work unless the variable is an OGLE star, in which case we use their period; Col. 7 is the median magnitude; Col. 8 is the peak-to-peak amplitude in the light curve; Col. 9 is the number of epochs; and Col. 10 is the classification of the variable. The numbers in parentheses indicate the uncertainty on the last decimal place of the period. ${ }^{(a)}$ Epochs, periods, mean magnitudes, amplitudes, and classifications taken from OGLE database; ${ }^{(b)}$ SPVSgr18550405-3028580; ${ }^{(c)}$ SPVSgr18550386-3028593; ${ }^{(d)}$ peak magnitude. 
Table A.1. continued.

\begin{tabular}{|c|c|c|c|c|c|c|c|c|c|}
\hline $\begin{array}{l}\text { Var } \\
\text { id } \\
(1)\end{array}$ & $\begin{array}{l}\text { Other } \\
\text { id } \\
(2)\end{array}$ & $\begin{array}{c}\text { RA } \\
\text { J2000 } \\
(3)\end{array}$ & $\begin{array}{c}\text { Dec } \\
\text { J2000 } \\
(4)\end{array}$ & $\begin{array}{c}\text { Epoch } \\
\text { HJD } \\
(5)\end{array}$ & $\begin{array}{c}P \\
\mathrm{~d} \\
(6) \\
\end{array}$ & $\begin{array}{c}I_{\text {median }} \\
\text { mag } \\
(7)\end{array}$ & $\begin{array}{c}A_{i^{\prime}+z^{\prime}} \\
\mathrm{mag} \\
(8)\end{array}$ & (9) & Type \\
\hline V253 & 37586 & $18: 55: 03.268$ & $-30: 28: 28.69$ & 2457206.6555 & $0.74391157(1160)$ & 17.32 & 0.29 & 17 & RR0 \\
\hline $\mathrm{V} 254$ & O16 & $18: 55: 02.808$ & $-30: 28: 49.69$ & 2456436.8969 & $0.747628(652)$ & 17.06 & 0.71 & 44 & RR0 \\
\hline V255 & O16; VC5 & $18: 55: 03.123$ & $-30: 28: 37.99$ & 2456765.8300 & $0.760088(674)$ & 16.48 & 0.19 & 44 & RR0 \\
\hline V256 & O16 & $18: 55: 03.730$ & $-30: 28: 44.94$ & 2456784.9179 & $14.771(25)$ & 14.72 & 0.71 & 44 & CWA \\
\hline V257 & - & $18: 55: 02.996$ & $-30: 28: 43.18$ & 2456823.8857 & $20.747(50)$ & 14.04 & 0.09 & 44 & SR \\
\hline $\mathrm{V} 258$ & - & $18: 55: 03.732$ & $-30: 28: 48.34$ & 2457224.5000 & $37.980(168)$ & 13.16 & 0.04 & 44 & SR \\
\hline V259 & - & $18: 55: 04.216$ & $-30: 28: 49.91$ & 2456464.9081 & $154(3)$ & 13.30 & 0.45 & 44 & SR \\
\hline V260 & - & $18: 55: 03.170$ & $-30: 28: 54.44$ & - & - & 12.76 & 0.06 & 44 & $\mathrm{~L}$ \\
\hline V261 & - & 18:55:03.399 & $-30: 28: 44.99$ & - & - & 13.78 & 0.07 & 44 & $\mathrm{~L}$ \\
\hline V262 & O16 & $18: 55: 01.876$ & $-30: 28: 43.79$ & - & - & 13.99 & 0.07 & 40 & $\mathrm{~L}$ \\
\hline V263 & $\mathrm{O} 16 ;^{c}$ & $18: 55: 03.877$ & $-30: 28: 59.31$ & - & - & 14.71 & 0.07 & 44 & $\mathrm{~L}$ \\
\hline V264 & - & $18: 55: 03.753$ & $-30: 28: 49.36$ & - & - & 13.87 & 0.08 & 44 & $\mathrm{~L}$ \\
\hline V265 & - & $18: 55: 02.987$ & $-30: 28: 51.96$ & - & - & 14.00 & 0.08 & 44 & $\mathrm{~L}$ \\
\hline V266 & - & $18: 55: 03.458$ & $-30: 28: 46.47$ & - & - & 14.04 & 0.08 & 44 & $\mathrm{~L}$ \\
\hline V267 & - & $18: 55: 03.463$ & $-30: 28: 45.05$ & - & - & 13.66 & 0.09 & 44 & $\mathrm{~L}$ \\
\hline V268 & - & $18: 55: 02.998$ & $-30: 28: 44.30$ & - & - & 13.79 & 0.09 & 44 & $\mathrm{~L}$ \\
\hline V269 & - & $18: 55: 03.712$ & $-30: 28: 43.08$ & - & - & 13.62 & 0.10 & 44 & $\mathrm{~L}$ \\
\hline V270 & O16 & $18: 55: 02.603$ & $-30: 28: 48.92$ & - & - & 13.70 & 0.11 & 44 & $\mathrm{~L}$ \\
\hline V271 & O16 & $18: 55: 04.004$ & $-30: 28: 37.05$ & - & - & 13.97 & 0.12 & 44 & $\mathrm{~L}$ \\
\hline $\mathrm{V} 272$ & - & $18: 55: 03.065$ & $-30: 28: 47.99$ & - & - & 14.25 & 0.05 & 44 & $\mathrm{~L}$ \\
\hline V273 & $\mathrm{O} 16$ & $18: 55: 03.708$ & $-30: 28: 35.30$ & - & - & 13.40 & 0.15 & 44 & $\mathrm{~L}$ \\
\hline V274 & O16 & $18: 55: 03.833$ & $-30: 29: 03.89$ & - & - & 13.47 & 0.08 & 44 & $\mathrm{~L}$ \\
\hline$V 275$ & 016 & $18: 55: 02.346$ & $-30: 28: 43.40$ & - & - & 13.67 & 0.16 & 44 & $\mathrm{~L}$ \\
\hline V276 & - & $18: 55: 03.659$ & $-30: 28: 50.52$ & - & - & 14.06 & 0.16 & 44 & $\mathrm{~L}$ \\
\hline V277 & - & $18: 55: 03.309$ & $-30: 28: 49.79$ & - & - & 13.71 & 0.19 & 44 & $\mathrm{~L}$ \\
\hline V278 & - & $18: 55: 04.070$ & $-30: 28: 49.79$ & - & - & 13.13 & 0.33 & 44 & $\mathrm{~L}$ \\
\hline V279 & O16 & $18: 55: 04.045$ & $-30: 28: 41.41$ & - & - & 13.42 & 0.34 & 44 & $\mathrm{~L}$ \\
\hline V280 & O16 & $18: 55: 03.692$ & $-30: 28: 52.01$ & - & - & 13.25 & 0.46 & 44 & $\mathrm{~L}$ \\
\hline V281 & - & $18: 55: 03.324$ & $-30: 28: 47.26$ & - & - & 16.71 & 0.45 & 44 & $\mathrm{NC}$ \\
\hline V282 & - & $18: 55: 03.010$ & $-30: 28: 35.82$ & - & - & 17.52 & 0.74 & 44 & $\mathrm{NC}$ \\
\hline V283 & - & $18: 55: 03.747$ & $-30: 28: 53.88$ & - & - & 16.97 & 0.76 & 44 & $\mathrm{NC}$ \\
\hline V284 & O16; VC32 & $18: 55: 03.367$ & $-30: 28: 37.97$ & - & - & 17.14 & 0.94 & 44 & $\mathrm{NC}$ \\
\hline V285 & O16; VC33 & $18: 55: 02.686$ & $-30: 28: 32.48$ & - & - & 17.52 & 0.55 & 44 & $\mathrm{NC}$ \\
\hline V286 & 016 & $18: 55: 03.536$ & $-30: 28: 51.06$ & - & - & $15.04^{d}$ & - & 43 & $\mathrm{NC}$ \\
\hline V287 & O16 & $18: 55: 03.373$ & $-30: 28: 35.86$ & - & - & - & - & 44 & $\mathrm{NC}$ \\
\hline V288 & - & $18: 55: 03.412$ & $-30: 28: 52.76$ & - & - & - & - & 44 & $\mathrm{NC}$ \\
\hline V289 & O16 & $18: 55: 02.904$ & $-30: 28: 39.89$ & - & - & - & - & 42 & $\mathrm{NC}$ \\
\hline V290 & O16 & $18: 55: 03.475$ & $-30: 29: 06.23$ & - & - & - & - & 39 & $\mathrm{NC}$ \\
\hline V291 & O16; VC27 & $18: 55: 03.258$ & $-30: 28: 41.09$ & - & - & - & - & 42 & $\mathrm{NC}$ \\
\hline
\end{tabular}



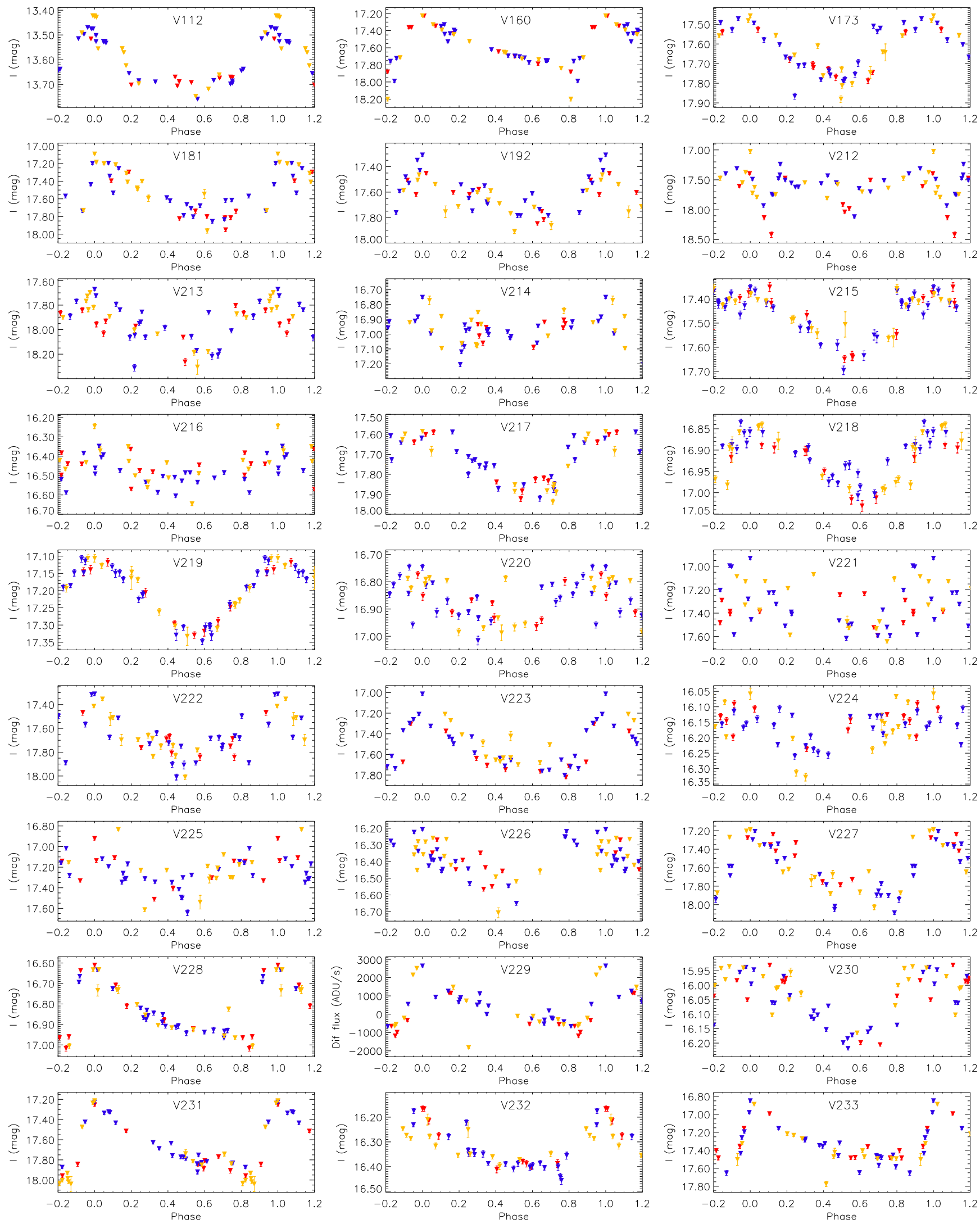

(a)

Fig. A.1. Light curves of the known and new variables discovered in globular cluster NGC 6715. Red, blue, and yellow triangles correspond to the data obtained during the years 2013,2014 , and 2015, respectively. For V229, V244, V246, V286-V291, we plot the quantity $f_{\text {diff }}(t) / p(t)$ since a reference flux is not available. 
A\&A 592, A120 (2016)
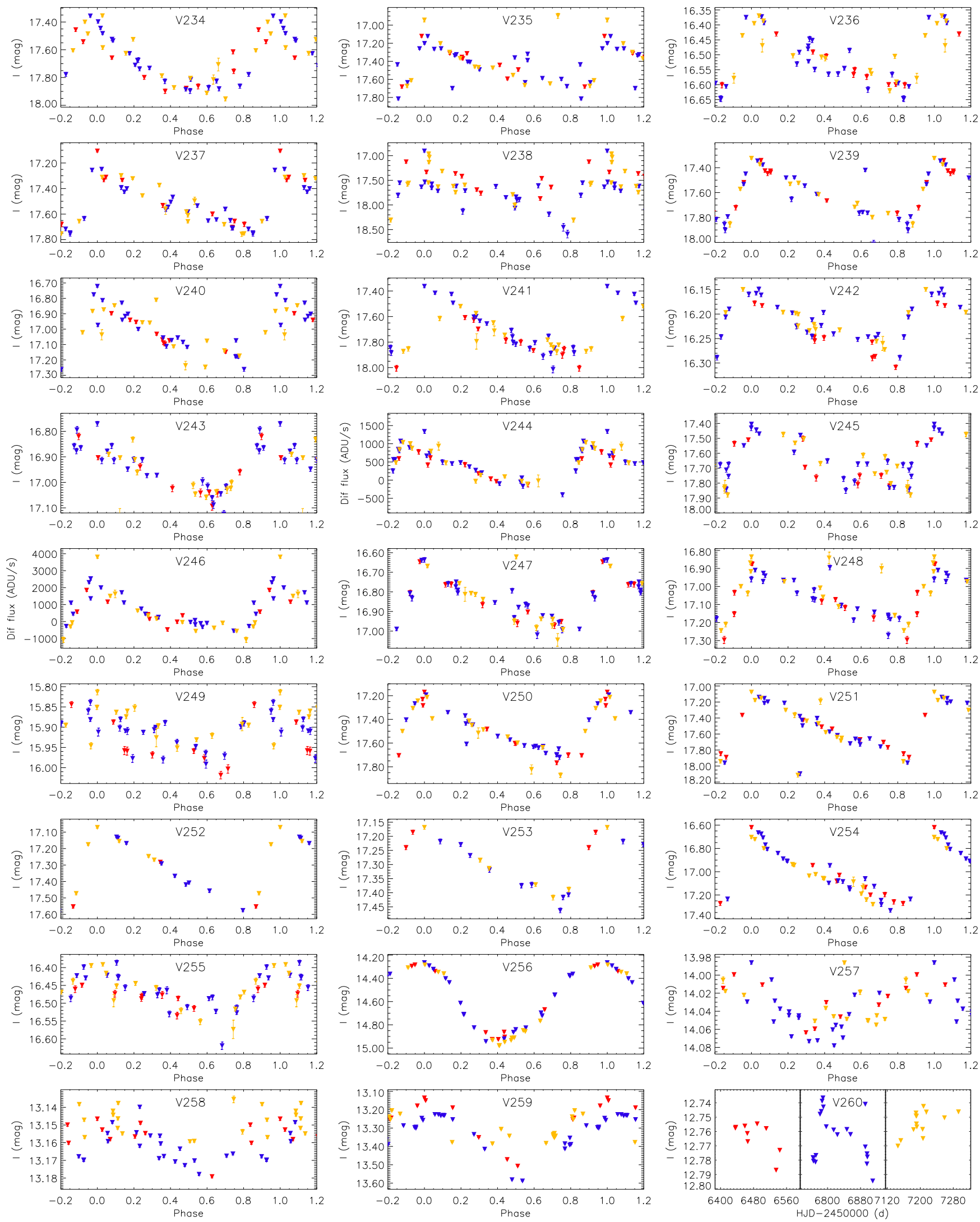

(b)

Fig. A.1. continued. 

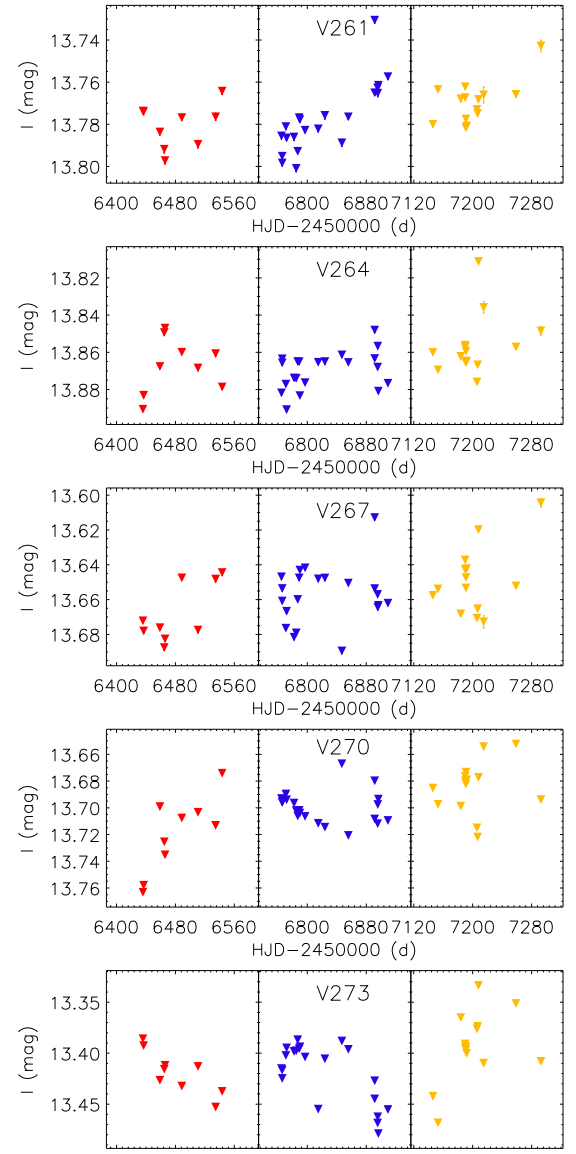

64006480656068006880712072007280
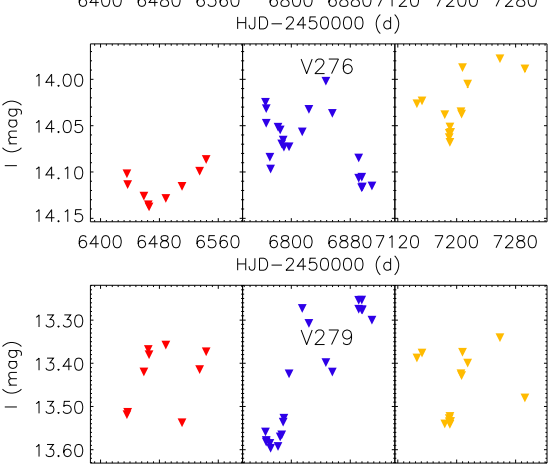

64006480656068006880712072007280

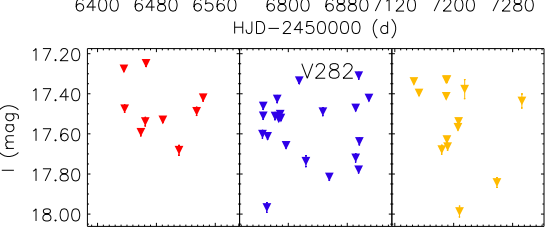

$640064806560 \quad 68006880712072007280$

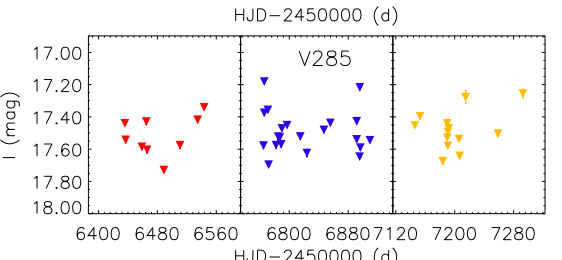

$6400 \quad 64806560 \quad 680068807120$
HJD -2450000 (d)
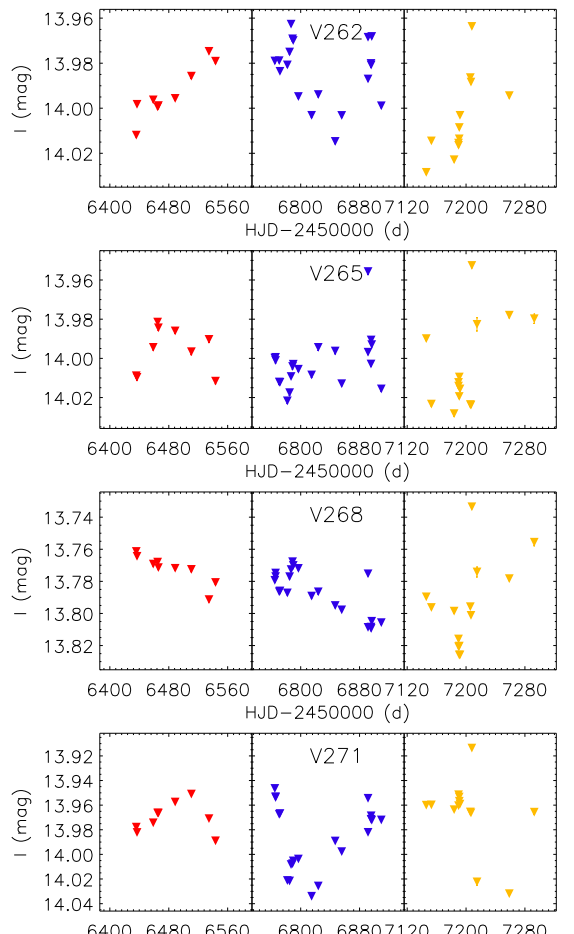

$640064806560 \quad 68006880712072007280$
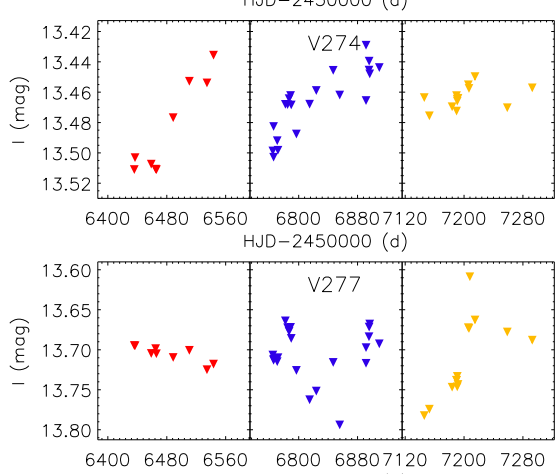

$6400 \quad 64806560 \quad 68006880712072007280$

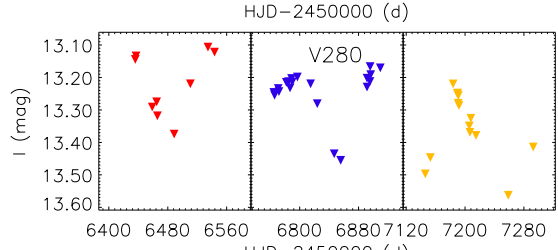

$640064806560 \quad 68006880712072007280$

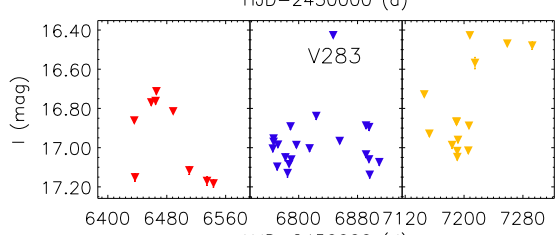

$640064806560 \quad 68006880712072007280$

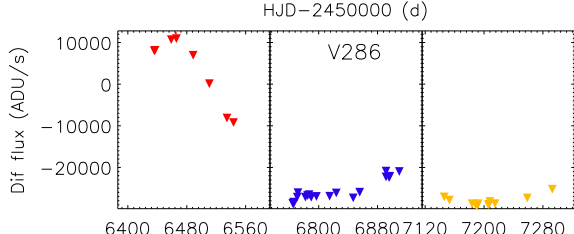

$640064806560 \quad 68006880712072007280$

(c)
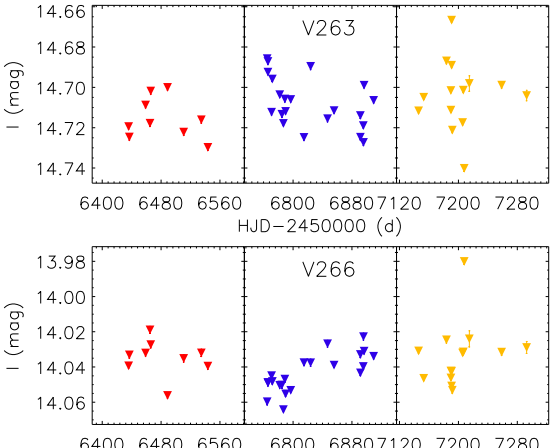

$640064806560 \quad 68006880712072007280$

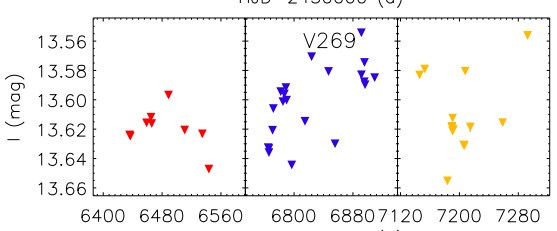
HJD -2450000 (d)
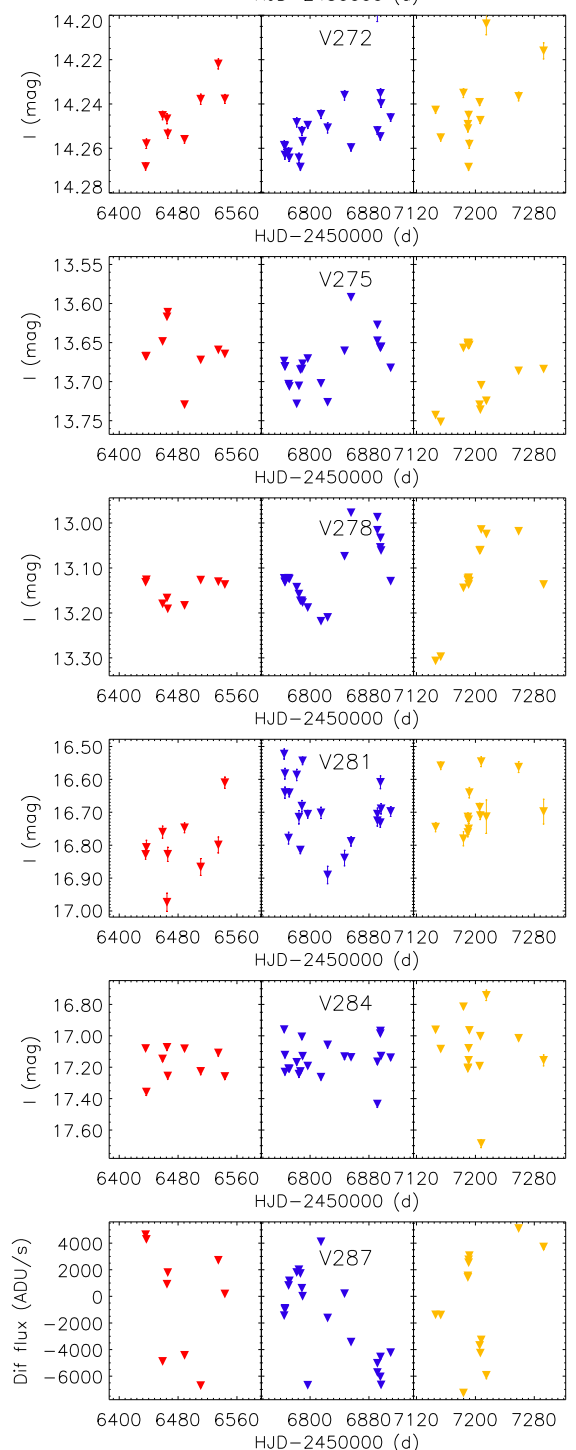

Fig. A.1. continued. 
A\&A 592, A120 (2016)
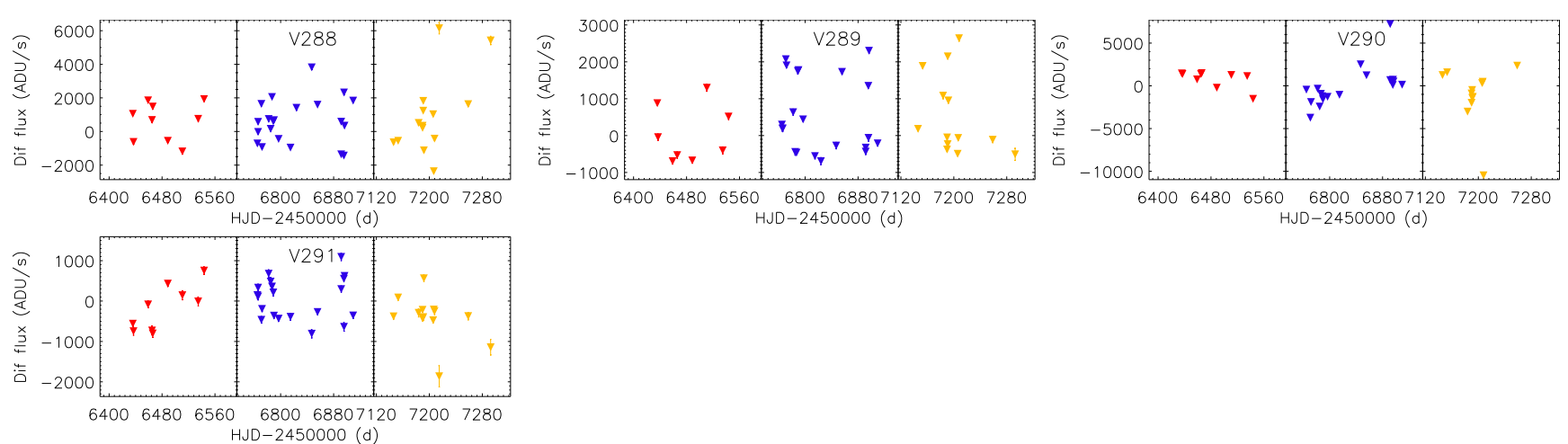

(d)

Fig. A.1. continued. 


\section{Appendix B: Cross-identification between variables}

Table B.1. Cross-identification between the RR Lyrae variables listed by the CVSGGC (Clement et al. 2001), the variable star candidates from Montiel \& Mighell (2010), and the OGLE RR Lyrae stars (Soszyński et al. 2014).

\begin{tabular}{|c|c|c|c|c|c|c|c|c|}
\hline $\begin{array}{c}\text { CVSGGC } \\
\text { id }\end{array}$ & $\begin{array}{c}\text { OGLE } \\
\text { id } \\
\text { (OGLE-BLG-) }\end{array}$ & $\begin{array}{c}\text { Montiel } \\
\text { id }\end{array}$ & $\begin{array}{c}\text { CVSGGC } \\
\text { id }\end{array}$ & $\begin{array}{l}\text { OGLE } \\
\text { id }\end{array}$ & $\begin{array}{l}\text { Montiel } \\
\text { id }\end{array}$ & $\begin{array}{c}\text { CVSGGC } \\
\text { id }\end{array}$ & $\begin{array}{l}\text { OGLE } \\
\text { id }\end{array}$ & $\begin{array}{c}\text { Montiel } \\
\text { id }\end{array}$ \\
\hline V3 & RRLYR-37571 & & V92 & RRLYR-37611 & & V186 & RRLYR-37546 & \\
\hline V4 & RRLYR-37540 & & V93 & RRLYR-37567 & & V188 & RRLYR-37584 & \\
\hline V5 & RRLYR-37518 & & V94 & RRLYR-37634 & & V192 & RRLYR-37568 & VC46 \\
\hline V6 & RRLYR-37655 & & V95 & RRLYR-37560 & VC13 & V193 & RRLYR-37605 & \\
\hline V7 & RRLYR-37620 & & V97 & RRLYR-37607 & & V194 & RRLYR-37574 & \\
\hline V12 & RRLYR-37511 & & V98 & RRLYR-37637 & & V227 & RRLYR-37582 & \\
\hline V15 & RRLYR-37649 & & V118 & RRLYR-37660 & & V228 & RRLYR-37575 & \\
\hline V28 & RRLYR-37623 & & V119 & RRLYR-37654 & & V229 & RRLYR-37597 & \\
\hline V29 & RRLYR-37519 & & V120 & RRLYR-37638 & & V233 & RRLYR-37591 & \\
\hline V31 & RRLYR-37528 & & V121 & RRLYR-37635 & & V236 & RRLYR-37585 & \\
\hline V32 & RRLYR-37514 & & V122 & RRLYR-37632 & & V237 & RRLYR-37570 & \\
\hline V33 & RRLYR-37631 & & V123 & RRLYR-37583 & & V240 & RRLYR-37576 & \\
\hline V34 & RRLYR-37541 & & V124 & RRLYR-37628 & & V244 & RRLYR-37581 & \\
\hline V35 & RRLYR-37533 & & V125 & RRLYR-37613 & & V246 & RRLYR-37573 & \\
\hline V36 & RRLYR-37648 & & V126 & RRLYR-37600 & & V250 & RRLYR-37590 & \\
\hline V37 & RRLYR-37617 & & V127 & RRLYR-37580 & $\mathrm{VC} 2$ & V251 & RRLYR-37579 & \\
\hline V38 & RRLYR-37538 & & V128 & RRLYR-37551 & & V252 & RRLYR-37593 & \\
\hline V39 & RRLYR-37527 & & V129 & RRLYR-37526 & VC17 & V253 & RRLYR-37586 & \\
\hline V40 & RRLYR-37543 & & V130 & RRLYR-37549 & & - & RRLYR-37578 & VC38 \\
\hline V41 & RRLYR-37647 & & V131 & RRLYR-37525 & & & & \\
\hline V42 & RRLYR-37626 & & V132 & RRLYR-37555 & & & & \\
\hline V43 & RRLYR-37516 & & V133 & RRLYR-37550 & & & & \\
\hline V44 & RRLYR-37599 & & V136 & RRLYR-37513 & & & & \\
\hline V45 & RRLYR-37646 & & V137 & RRLYR-37612 & & & & \\
\hline V46 & RRLYR-37552 & VC44 & V138 & RRLYR-37610 & & & & \\
\hline V47 & RRLYR-37554 & & V139 & RRLYR-37596 & & & & \\
\hline V48 & RRLYR-37659 & & V140 & RRLYR-37572 & & & & \\
\hline V49 & RRLYR-37532 & & V141 & RRLYR-37558 & & & & \\
\hline V50 & RRLYR-37640 & & V142 & RRLYR-37545 & VC15 & & & \\
\hline V51 & RRLYR-37657 & & V143 & RRLYR-37534 & & & & \\
\hline V52 & RRLYR-37636 & & V147 & RRLYR-37602 & & & & \\
\hline V54 & RRLYR-37521 & & V148 & - & VC45 & & & \\
\hline V55 & RRLYR-37651 & & V151 & RRLYR-37517 & & & & \\
\hline V57 & RRLYR-37666 & & V152 & RRLYR-37615 & & & & \\
\hline V58 & RRLYR-37630 & & V158 & RRLYR-37539 & & & & \\
\hline V59 & RRLYR-37512 & & V159 & RRLYR-37589 & & & & \\
\hline V60 & RRLYR-37509 & & V160 & RRLYR-37595 & VC34 & & & \\
\hline V61 & RRLYR-37547 & & V161 & RRLYR-37616 & & & & \\
\hline V62 & RRLYR-37531 & & V162 & RRLYR-37592 & VC11 & & & \\
\hline V63 & RRLYR-37553 & & V163 & RRLYR-37604 & VC12 & & & \\
\hline V67 & RRLYR-37587 & & V164 & RRLYR-37564 & VC14 & & & \\
\hline V69 & RRLYR-37505 & & V165 & RRLYR-37609 & & & & \\
\hline V74 & RRLYR-37645 & & V168 & RRLYR-37619 & & & & \\
\hline V76 & RRLYR-37529 & $\mathrm{VC} 47$ & V172 & RRLYR-37614 & & & & \\
\hline V77 & RRLYR-37524 & & V174 & RRLYR-37608 & & & & \\
\hline V78 & RRLYR-37629 & & V176 & RRLYR-37618 & & & & \\
\hline V80 & RRLYR-37624 & & V177 & RRLYR-37556 & & & & \\
\hline V82 & RRLYR-37548 & & V178 & RRLYR-37562 & & & & \\
\hline V83 & RRLYR-37522 & & V179 & RRLYR-37557 & VC18 & & & \\
\hline V84 & RRLYR-37577 & & V180 & RRLYR-37588 & & & & \\
\hline V85 & RRLYR-37569 & & V181 & - & VC28 & & & \\
\hline V87 & RRLYR-37641 & & V182 & RRLYR-37561 & & & & \\
\hline V88 & RRLYR-37515 & & V183 & RRLYR-37603 & & & & \\
\hline V89 & RRLYR-37530 & & V184 & RRLYR-37559 & & & & \\
\hline V90 & RRLYR-37622 & & V185 & RRLYR-37563 & & & & \\
\hline
\end{tabular}

